OPEN ACCESS

Edited by:

Edward S. Ruthazer

McGill University, Canada

Reviewed by:

Grant Robert Gordon,

University of Calgary, Canada

Gertrudis Perea,

Cajal Institute (CSIC), Spain

${ }^{*}$ Correspondence:

Krissy A. Lyon

klyon@salk.edu

Nicola J. Allen

nallen@salk.edu

Received: 30 September 2021 Accepted: 05 November 2021

Published: 04 January 2022

Citation:

Lyon KA and Allen NJ (2022)

From Synapses to Circuits,

Astrocytes Regulate Behavior.

Front. Neural Circuits 15:786293.

doi: 10.3389/fncir.2021.786293

\section{From Synapses to Circuits, Astrocytes Regulate Behavior}

\author{
Krissy A. Lyon* and Nicola J. Allen* \\ Molecular Neurobiology Laboratory, The Salk Institute for Biological Studies, La Jolla, CA, United States
}

Astrocytes are non-neuronal cells that regulate synapses, neuronal circuits, and behavior. Astrocytes ensheath neuronal synapses to form the tripartite synapse where astrocytes influence synapse formation, function, and plasticity. Beyond the synapse, recent research has revealed that astrocyte influences on the nervous system extend to the modulation of neuronal circuitry and behavior. Here we review recent findings on the active role of astrocytes in behavioral modulation with a focus on in vivo studies, primarily in mice. Using tools to acutely manipulate astrocytes, such as optogenetics or chemogenetics, studies reviewed here have demonstrated a causal role for astrocytes in sleep, memory, sensorimotor behaviors, feeding, fear, anxiety, and cognitive processes like attention and behavioral flexibility. Current tools and future directions for astrocyte-specific manipulation, including methods for probing astrocyte heterogeneity, are discussed. Understanding the contribution of astrocytes to neuronal circuit activity and organismal behavior will be critical toward understanding how nervous system function gives rise to behavior.

Keywords: astrocyte, behavior, chemogenetic, optogenetic, GPCR

\section{INTRODUCTION}

A major goal in neuroscience is to understand how neuronal circuitry maps onto specific physiological functions and behaviors. Neural circuits are recognized as the basis of nervous system function, therefore neuroscience research has largely focused on neurons from the level of the synapse to brain-wide circuits (Tosches, 2017). Yet, the contributions of astrocytes, key players in brain function, have long been overlooked. Astrocytes are glial cells that tile throughout the brain ensheathing neuronal synapses to form the "tripartite synapse," in which neurons and astrocytes bidirectionally communicate (Araque et al., 1999; Allen and Barres, 2009). The functions of astrocytes at the synapse include clearance of excess neurotransmitter, maintenance of ion homeostasis, and release of neuroactive factors influencing synapse development and function (Allen and Barres, 2009; Murphy-Royal et al., 2017; Farhy-Tselnicker and Allen, 2018). Bidirectional astrocyte-neuron communication includes astrocyte response to synaptic activity via neurotransmitter receptors (Porter and McCarthy, 1997; Kofuji and Araque, 2021) and astrocytic release of factors. Unlike neurons, astrocytes do not fire action potentials, but are capable of release of gliotransmitters (Bezzi and Volterra, 2001; Bezzi et al., 2004; Fiacco and McCarthy, 2004; Jourdain et al., 2007; Navarrete et al., 2013; Araque et al., 2014; Harada et al., 2015) such as ATP (Guthrie et al., 1999; Gourine et al., 2010) and D-serine (Wolosker et al., 1999; Yang et al., 2003; 
Henneberger and Rusakov, 2010) which can modulate neuronal activity. While the importance of astrocytes for proper synapse formation, function, and plasticity is now appreciated (FarhyTselnicker and Allen, 2018; Durkee and Araque, 2019; Sancho et al., 2021; Shan et al., 2021; Wahis et al., 2021b), many questions remain about the capacity of astrocytes to influence behavior.

In this review, we will focus on recent findings of astrocyte influence on behavior including sleep, memory, sensorimotor activity, feeding behavior, fear, anxiety, and additional cognitive processes. Prior to the advent of optogenetic and chemogenetic tools, the study of astrocyte regulation of behavior was hindered by the lack of tools to specifically manipulate astrocytes without also targeting neurons. For example, pharmacological approaches, such as thapsigargin, a SERCA pump inhibitor used to elevate intracellular calcium concentrations (Jackson et al., 1988), might target all cell populations. We will discuss current tools to selectively manipulate astrocytes as well as future directions for further delineation of astrocyte regulation of behavior, especially as relates to understanding astrocyte function based on anatomical location or astrocyte subtypes. Among the extensive literature, this review will highlight the most recent research, particularly in vivo studies, of astrocyte influence on behavior in rodents. In vitro studies remain critical for elucidation of molecular and cellular mechanisms, but in vivo experiments are necessary for understanding the role of astrocytes in whole organismal behavior. Likewise, in vivo experiments can address the effects of sensory input and behavioral state on astrocytes themselves (Nimmerjahn, 2009). In vitro astrocyte manipulations have been reviewed elsewhere (Bang et al., 2016) as have recent findings including C. elegans, D. rerio, and D. melanogaster (Nagai et al., 2021a).

\section{Physiologically Relevant Tools to Manipulate Astrocyte Activity}

Astrocyte excitability is primarily mediated through calcium signaling (Agulhon et al., 2008; Nimmerjahn, 2009; Zorec et al., 2012) which is complex and varies in duration, amplitude, event rate, and cellular localization of calcium events (Shigetomi et al., 2008, 2010; Bazargani and Attwell, 2016; Wang et al., 2019). Astrocytes display dynamic intracellular calcium signaling which can be spontaneous or triggered by neuronal activity (Cornell-Bell et al., 1990; Nett et al., 2002; Khakh and McCarthy, 2015; Bazargani and Attwell, 2016). One of the main communication pathways between neurons and astrocytes is through G-protein coupled receptors (GPCRs) expressed by astrocytes (Figure 1A; Neves et al., 2002; Kofuji and Araque, 2021). GPCRs interact with heterotrimeric G proteins alpha $(\alpha)$, beta $(\beta)$, and gamma $(\gamma)$ (Figure $B$ ). Following GPCR activation, the alpha subunit, subdivided into Gq, Gi, and Gs families, dissociates from the beta and gamma proteins leading to downstream signaling cascades including the phospholipase C (PLC)/inositol 1,4,5-triphosphate (IP3) pathway which results in calcium release from the endoplasmic reticulum (Figure 1B; Yu et al., 2020; Kofuji and Araque, 2021). In vivo fluorescent calcium imaging revealed correlations of astrocyte calcium activity with visual responses
(Schummers et al., 2008), locomotion (Dombeck et al., 2007; Nimmerjahn et al., 2009) and cortical oscillations (Poskanzer and Yuste, 2016) indicating a link between astrocyte calcium activity, neuronal circuits, and behavior. While astrocyte intracellular calcium signaling is critical to astrocyte-neuron communication, the functional relevance of different features of calcium signaling remains a topic of exploration (Bazargani and Attwell, 2016; Guerra-Gomes et al., 2017).

Many recent studies pair in vivo calcium imaging with effector tools to simultaneously image astrocyte or neuron calcium activity alongside astrocyte-specific perturbations (see Table 1 for summary of tools) (Gourine et al., 2010; Chen et al., 2016; Asrican et al., 2020; Corkrum et al., 2020; Ung et al., 2020; Vaidyanathan et al., 2021). For cell type specificity, astrocytes are primarily targeted by expressing effectors under the control of Aldh1l1 (Tsai et al., 2012), GFAP (Lee et al., 2008), or the shortened GfaABC1D promoters (Shigetomi et al., 2013; Figure 2A), either through viral vector delivery or in transgenic rodents. Both optogenetic and chemogenetic tools have been applied in astrocytes but experimental manipulation of astrocytes comes with additional caveats (Mu et al., 2019). Astrocytes are not neurons and tools designed for use in neurons must be carefully characterized in astrocytes before assessing behavioral changes (Figueiredo et al., 2014; McNeill et al., 2021). For example, the light-sensitive cation channel channelrhodopsin is widely used to activate neurons through the generation of action potentials (Boyden et al., 2005). Yet, studies have employed channelrhodopsin in astrocytes which do not fire action potentials. Stimulation of channelrhodopsin in astrocytes is used to increase intracellular calcium, but this also leads to an increase in extracellular potassium (Octeau et al., 2019). While the mechanism of increased potassium is unknown, such increases could impact neuronal firing and confound behavioral results (Octeau et al., 2019; Rasmussen et al., 2019) highlighting the importance of careful characterization of tools in astrocytes. Given the highly prevalent expression of GPCRs in astrocytes, tools targeting GPCR signaling pathways may be more physiologically relevant. However, it is important to note that Gicoupled DREADDs (Designer Receptors Exclusively Activated by Designer Drugs) (Alexander et al., 2009; Roth, 2016) employed to inhibit neurons have the opposite effect on astrocytes and increase calcium activity and promote gliotransmitter release (Chai et al., 2017; Durkee et al., 2019; Figure 1). Lastly, astrocytes also contact other astrocytes via gap junctions (Rose and Ransom, 1997), so studies aiming to manipulate subsets of astrocytes must also consider the interconnectedness of astrocytes (McNeill et al., 2021).

\section{Astrocyte Regulation of Behavior}

Early evidence for astrocyte regulation of behavior emerged when Halassa et al. (2009) manipulated astrocyte function and observed altered sleep (discussed below in Sleep section). Next, chemosensitive hindbrain astrocytes were shown to modulate respiration in rats (Gourine et al., 2010). In response to decreases in $\mathrm{pH}$, corresponding to elevated blood $\mathrm{CO}_{2}$ levels, astrocyte intracellular calcium levels rise leading to increased astrocytic ATP release, chemoreceptor neuron activation, and increased adaptive breathing revealing a role for astrocytes in a 


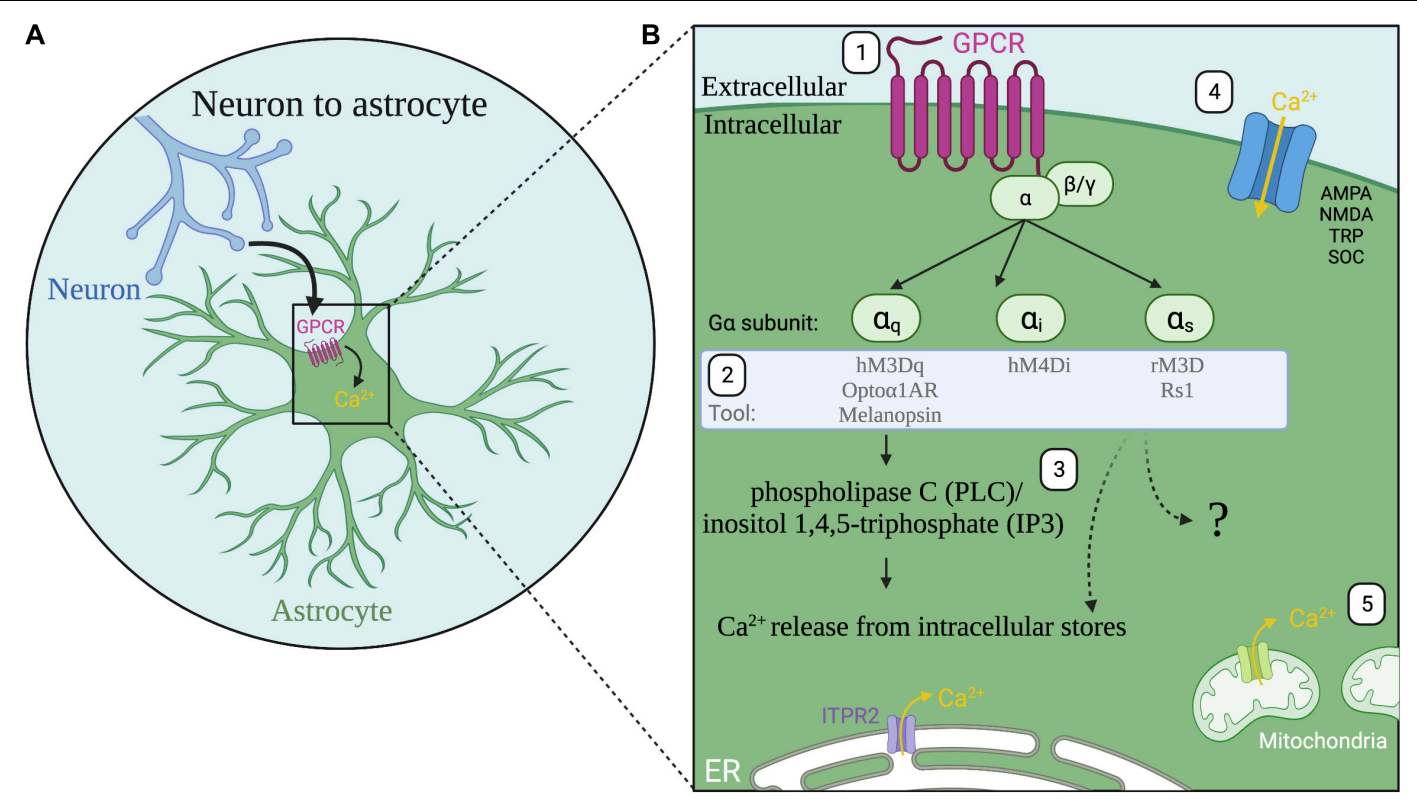

FIGURE 1 | Astrocyte G-protein coupled receptor (GPCR) signaling. (A) A major communication pathway from neurons to astrocytes occurs through GPCRs expressed by astrocytes. (B) (1) Activation of astrocyte GPCRs (magenta) activate alpha subunits Gaq, Gai, or Gas. (2) Tools for astrocyte manipulation target these pathways. (3) Following Gq GPCR activation, the phospholipase C (PLC)/inositol 1,4,5-triphosphate (IP3) pathway and ITPR2 receptor activation induces release of calcium $\left(\mathrm{Ca}^{2+}\right.$, yellow) from the endoplasmic reticulum (ER, white). All three G protein signaling pathways are known to increase calcium in astrocytes though the intracellular signaling pathways are not fully resolved (question mark). (4) Additional sources of calcium include calcium influx through ionotropic glutamate receptors $\alpha$-amino-3-hydroxy-5-methyl-4-isoxazolepropionic acid receptor (AMPA) and N-methyl-d-aspartate receptor (NMDA); transient receptor potential (TRP) channels; store-operated calcium channels (SOC); and reversed operation of the $\mathrm{Na}^{+} / \mathrm{Ca}^{2+}$ exchanger (not shown). (5) Calcium is also released from mitochondria.

critical homeostatic reflex. Other studies showed that activation of astrocyte Gq-GPCR signaling using hM3Dq DREADD increased heart rate and blood pressure, while decreasing body temperature, indicating astrocyte involvement in additional homeostatic processes (Agulhon et al., 2013). Over the past decade, a role for astrocytes in behavior modulation has become increasingly clear.

\section{Sleep}

Based upon his histological and morphological studies, Ramon y Cajal hypothesized that astrocytes control the switch from wakefulness to sleep (Garcia-Marin et al., 2007). Modern tools for astrocyte-specific manipulation have recently allowed researchers to probe neuron-astrocyte interactions in the regulation of sleep and wake in vivo. Using a mouse line expressing a dominant negative SNARE protein in astrocytes (DN-SNARE) (Pascual et al., 2005; Fujita et al., 2014) that alters astrocyte vesicular trafficking and release, Halassa et al. (2009) demonstrated a role for astrocytes in sleep pressure, or the drive to sleep following wakefulness. This was further shown to be in an adenosine A1 receptor-dependent manner linking astrocyte-derived adenosine to sleep regulation. Later studies outlined below employed optogenetic activation of astrocytes to acutely test a role in sleep.

Neuronal activity changes in different phases of sleep. During rapid eye movement (REM) sleep neural oscillations are fast and desynchronized while neuronal activity in nonREM (NREM) sleep is synchronized with high-amplitude, lowfrequency oscillations also referred to as slow wave sleep (Maquet,
2001). Channelrhodopsin activation of astrocytes in the anterior cingulate cortex of mice reduces NREM sleep in the light period (Yamashita et al., 2014) while activation of posterior hypothalamus astrocytes increases REM and NREM sleep in the dark period (Pelluru et al., 2016), indicating possible regionspecific, or circadian time-dependent, roles for astrocytes in the regulation of sleep. Indeed, suprachiasmatic nucleus astrocytes display increased calcium signaling at night (Brancaccio et al., 2017) and can drive circadian behavior in mice through glutamate release (Brancaccio et al., 2019). Lifelong astrocytespecific knockout of connexin 43, a gap junction component, also increases sleep in the dark period by silencing orexin wake neurons (Clasadonte et al., 2017). In vivo two-photon calcium imaging allowed researchers to correlate astrocyte calcium activity with features of sleep. For example, astrocyte calcium activity increases with sleep need in D. melanogaster (Blum et al., 2021). Additionally, increases in cortical astrocyte calcium precede shifts to slow wave activity, or the highly synchronized firing of neurons, in mice (Poskanzer and Yuste, 2016). While this latter study was conducted in anesthetized mice, this type of synchronized neuronal firing is typically indicative of slow wave sleep. Increasing astrocyte calcium activity through optogenetic activation shifted the local neuronal network to the synchronized state, suggesting an active role for astrocytes in cortical circuit dynamics (Poskanzer and Yuste, 2016).

Recently, calcium imaging in awake-behaving mice has enabled the study of natural sleep (Bojarskaite et al., 2020; Vaidyanathan et al., 2021). Tracking cortical astrocyte calcium 
TABLE 1 | Tools used to probe astrocyte influence on behavior.

\begin{tabular}{|c|c|c|c|}
\hline Tool & Description & Stimulus/ligand & Effect in astrocytes \\
\hline \multicolumn{4}{|l|}{ Optogenetics } \\
\hline Channelrhodopsin & Light-sensitive cation channel & Blue light & $\begin{array}{l}\text { Increases intracellular } \mathrm{Ca}^{2+} \text { in astrocytes Gourine et al. (2010) but also } \\
\text { increases extracellular } \mathrm{K}+\text { Octeau et al. (2019) }\end{array}$ \\
\hline Archaerhodopsin & Light-sensitive outward proton pump & Green-yellow light & Increases intracellular $\mathrm{Ca}^{2+}$ in astrocytes Poskanzer and Yuste (2016) \\
\hline Opto $\alpha 1 A R$ & Light-sensitive Gq-coupled receptor & Blue light & $\begin{array}{l}\text { Increases intracellular } \mathrm{Ca}^{2+} \text { in astrocytes Adamsky et al. (2018), for } \\
\text { mouse line see Iwai et al. (2021) }\end{array}$ \\
\hline Melanopsin (Opn4) & $\mathrm{G} \alpha_{q-11}$-coupled photopigment & Blue light & Increases intracellular $\mathrm{Ca}^{2+}$ in astrocytes Mederos et al. (2019) \\
\hline Mlc1-bPAC & $\begin{array}{l}\text { Photoactivated cyclase from Beggiatoa } \\
\text { bacterium }\end{array}$ & Blue light & Elevates astrocyte cAMP Zhou et al. (2021) \\
\hline \multicolumn{4}{|l|}{ Chemogenetics } \\
\hline hM3Dq & Gq-GPCR coupled DREADD & $\mathrm{CNO}$ & Increases intracellular $\mathrm{Ca}^{2+}$ in astrocytes Agulhon et al. (2013) \\
\hline hM4Di & Gi-GPCR coupled DREADD & $\mathrm{CNO}$ & $\begin{array}{l}\text { Increases intracellular } \mathrm{Ca}^{2+} \text { in astrocytes Chai et al. (2017), Durkee et al. } \\
\text { (2019) }\end{array}$ \\
\hline rM3D & Gs-GPCR coupled DREADD & $\mathrm{CNO}$ & Increases intracellular $\mathrm{Ca}^{2+}$ in astrocytes Chai et al. (2017) \\
\hline Rs1 & $\begin{array}{l}\text { Gs-GPCR coupled mutated serotonin } \\
\text { receptor }\end{array}$ & GR-125487 & $\begin{array}{l}\text { Increased cAMP in cultured astrocytes; Some ligand-independent } \\
\text { constitutive Gs-coupled activity Orr et al. (2015) }\end{array}$ \\
\hline \multicolumn{4}{|l|}{ Other } \\
\hline Itpr2-/- mouse line & $\begin{array}{l}\text { Knockout of } \mathrm{Ca}^{2+} \text { channel activated by } \\
\text { inositol trisphosphate }\end{array}$ & NA & Reduces GPCR mediated $\mathrm{Ca}^{2+}$ elevations Petravicz et al. (2008) \\
\hline DN-SNARE mouse line & $\begin{array}{l}\text { Dominant-negative domain of vesicular } \\
\text { SNARE }\end{array}$ & & $\begin{array}{l}\text { Alters astrocyte vesicular trafficking and release Pascual et al. (2005) but } \\
\text { see also Fujita et al. (2014) }\end{array}$ \\
\hline VIPP mouse line & $\begin{array}{l}\text { Overexpression of venus tagged } \\
\text { Ins }(1,4,5) \mathrm{P}_{3} 5^{\prime} \text { phosphatase (IPP) }\end{array}$ & & Metabolizes IP3, reduced $\mathrm{Ca}^{2+}$ signaling in astrocytes Foley et al. (2017) \\
\hline p130PH & $\begin{array}{l}\text { Overexpression of domain of } \\
\text { phospholipase C (PLC)-like protein p130 }\end{array}$ & & $\begin{array}{l}\text { Buffers cytosolic IP3 to inhibit release of Ca2 + from internal stores Xie } \\
\text { et al. (2010) }\end{array}$ \\
\hline ißARK & $\begin{array}{l}\text { RGS domain of } \beta \text {-adrenergic receptor } \\
\text { kinase } 1\end{array}$ & & $\begin{array}{l}\text { Sequesters Gaq-GTP, reducing Gq GPCR Ca }{ }^{2+} \text { signaling Nagai et al. } \\
\text { (2021b) }\end{array}$ \\
\hline CalEx & $\begin{array}{l}\text { Modified human plasma membrane } \mathrm{Ca}^{2+} \\
\text { pump PMCA2 }\end{array}$ & & Reduces astrocyte $\mathrm{Ca}^{2+}$ signaling Yu et al. (2018) \\
\hline Calcium indicators & $\begin{array}{l}\text { Typically fluorescence emission following } \\
\mathrm{Ca}^{2+} \text { binding }\end{array}$ & & Not a manipulation but a readout for calcium activity \\
\hline
\end{tabular}

Description of tools used to manipulate astrocytes, including details on astrocyte-specific effects.

activity across sleep and wake, Bojarskaite et al. (2020) found astrocyte calcium activity is lowest during sleep, increases during wake activities including whisking and locomotion, and is highest in the transition from sleep to wake. In this same study, Itpr2 (alias Ip3r2) knockout mice, which have attenuated astrocyte calcium signaling throughout the brain (Petravicz et al., 2008), did not show increased calcium upon awakening and had disrupted slow wave sleep (NREM and intermediate state sleep) linking astrocyte calcium activity to slow wave activity in natural sleep. Earlier studies found that mice with overexpression of Ins $(1,4,5) \mathrm{P}_{3} 5^{\prime}$-phosphatase (IPP) in astrocytes (Table 1), had attenuated calcium signaling in hippocampal astrocytes but largely normal calcium signaling in the cortex and normal NREM sleep (Foley et al., 2017), potentially indicating a specific role for cortical astrocytes in NREM sleep regulation.

Studies to increase astrocyte intracellular calcium using chemogenetic activation of cortical astrocyte Gi-GPCR signaling showed increased slow wave activity, whereas activation of the Gq-GPCR pathway disrupted sleep-wake transitions (Vaidyanathan et al., 2021). Thus, astrocytes regulate distinct features of natural sleep through separable signaling pathways. As noted above, both Gi and Gq signaling increase intracellular calcium in astrocytes (Mariotti et al., 2016; Durkee et al., 2019) thus this study shows that two different GPCR signaling pathways that both lead to increased calcium can lead to different behavioral outputs (Vaidyanathan et al., 2021). Existing evidence for astrocyte regulation of sleep primarily points to a role in the regulation of slow wave/NREM sleep. Future studies may continue to parse how distinct astrocyte calcium signaling pathways, and specific elements of calcium signaling such as cellular localization, duration, and amplitude (Wang et al., 2019), regulate different features of sleep. Beyond regulation of sleep-wake states, astrocytes promote solute clearance from the brain during sleep (Xie et al., 2013; Haydon, 2017) and astrocyte influences likely extend to the memory consolidation role of sleep, further discussed below.

\section{Memory}

Changes in sleep have the potential to impact other behaviors as memory consolidation is considered one important function of sleep (Stickgold, 2005; Abel and Klann, 2013; Klinzing et al., 2019). In discussing astrocytes and memory, we will briefly focus on known roles for astrocytes in both sleep and memory and then focus solely on memory. As discussed above, Itpr 2 knockout mice have blunted astrocyte calcium signaling and altered NREM sleep (Bojarskaite et al., 2020). Pinto-Duarte et al. (2019) found 


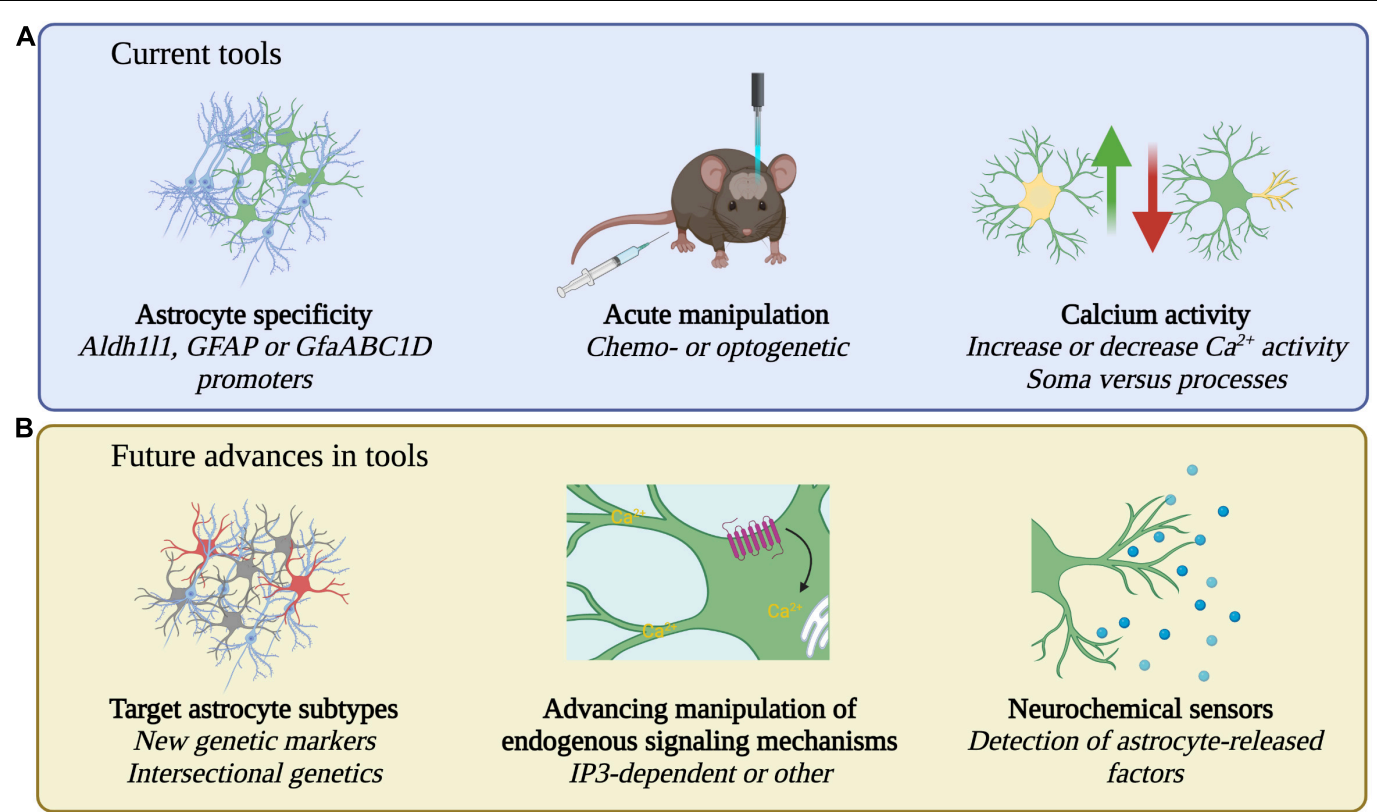

FIGURE 2 | Current and future tools for the study of astrocytes. (A) Current tools employ Aldh1ll1, GFAP, or GfaABC1D promoters to specifically target astrocytes. Acute manipulations including chemo- or optogenetic approaches and bidirectional increase or decrease of calcium activity, in soma or processes, are used to probe causal relationships between astrocytes and behavior. (B) Future research may identify new genetic markers for astrocytes, strategies for subtype specific manipulation, manipulations that target additional endogenous signaling pathways, and may detect astrocyte released factors with neurochemical sensors.

Itpr 2 knockout mice also have deficient remote memory recall but unaffected learning and short-term memory. While brainwide reduction of astrocyte calcium signaling disrupted NREM sleep and remote memory in separate studies, elevated cortical astrocyte calcium signaling increased delta oscillations which are associated with the weakening of memories (Vaidyanathan et al., 2021) indicating that astrocytes may influence both the strengthening and weakening of memories. While those studies did not test both sleep and memory regulation by astrocytes, Halassa et al. (2009) found impaired novel objection recognition following sleep deprivation in wildtype mice but DN-SNARE mice did not display this deficit. Thus, gliotransmission may regulate memory deficits following sleep deprivation.

Astrocyte secreted proteins and gliotransmitters shape neuronal plasticity (Santello et al., 2019; Sancho et al., 2021). Synaptic plasticity, including long term potentiation, underlies memory and astrocyte-released factors involved in these processes include D-serine (Yang et al., 2003; Panatier et al., 2006; Henneberger and Rusakov, 2010) and ATP/adenosine (Florian et al., 2011). Mice lacking these factors or their release from astrocytes display altered memory (Halassa et al., 2009; Florian et al., 2011; Lee et al., 2014). The contributions of astrocyte secreted factors and gliotransmission to memory are reviewed in detail elsewhere (Ben Achour and Pascual, 2012; De Pitta et al., 2016). Here, we will focus on recent work with acute manipulation of astrocytes, using optogenetic and chemogenetic tools, to probe astrocyte regulation of memory across phases of acquisition, consolidation, and retrieval. Different types of memory such as spatial memory or long-term memory can be tested in rodents and well-validated assays include the Y- and
T-mazes, novel object recognition and placement tests, Barnes maze, Morris water maze, contextual fear conditioning, and other cued memory assays (Swonger and Rech, 1972; Barnes, 1979; Vorhees and Williams, 2014; Wolf et al., 2016).

Many studies have used activation of the Gq-GPCR signaling pathway to test the role of astrocytes in memory. Chemogenetic activation of Gq-GPCR signaling in hippocampal astrocytes improved spatial memory and increased freezing in a contextual fear conditioning assay (Adamsky et al., 2018). Importantly, this Gq-GPCR activation was sufficient to generate de novo long term potentiation of CA3 to CA1 synapses thought to underly memory formation (Adamsky et al., 2018). Auditorycued memory was not affected indicating the engagement of hippocampal astrocyte Gq signaling in select types of memory. Further, increased freezing depended upon $\mathrm{Gq}$ activation during memory acquisition or early consolidation but was not affected by activation during memory recall (Adamsky et al., 2018) indicating a role for astrocyte Gq signaling in different phases of memory.

In the anterior cingulate cortex, Gq activation of astrocytes by the light inducible Gq-GPCR, Opto $\alpha 1$ AR (Table 1), increased long-term object recognition but did not affect Y-maze or novel object recognition assays (Iwai et al., 2021), suggesting a role for cortical astrocytes in long-term memory but not shortterm memory. In the prefrontal cortex (PFC), $\mathrm{GABA}_{\mathrm{B}}$ receptor knockout in astrocytes reduces low-gamma oscillation power and decreases performance in the T-maze (Mederos et al., 2021). Stimulation of a melanopsin GPCR tool coupled to $\mathrm{Gq}_{11}$ (Mederos et al., 2019; Table 1) in astrocytes rescues this deficit while $\mathrm{Gq}_{11}$ activation in PFC astrocytes in wildtype mice 


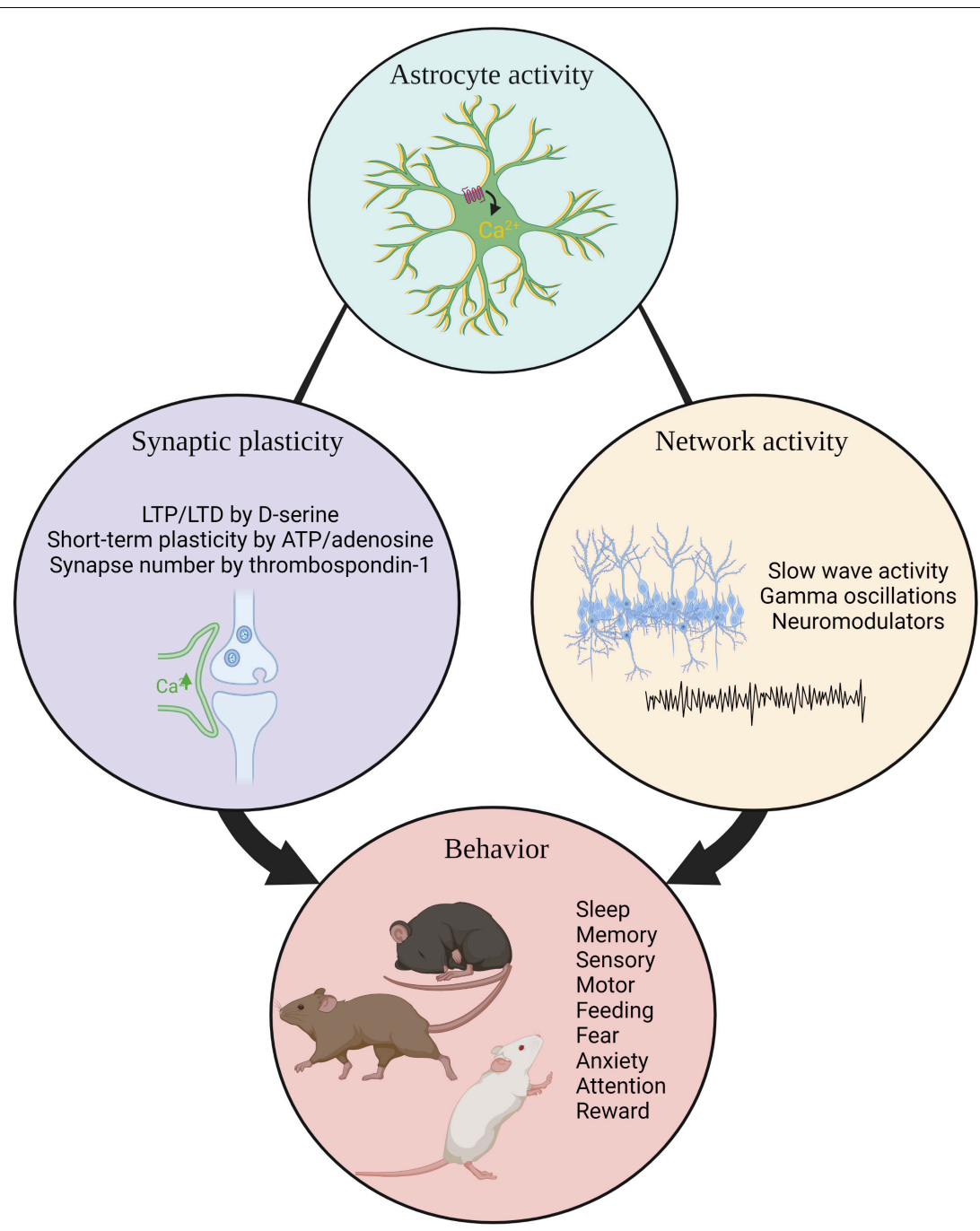

FIGURE 3 | Summary. Astrocyte activity influences synaptic plasticity, neuronal network activity, and organismal behavior. LTP, long term potentiation.; LTD, long term potentiation.

increases low-gamma oscillation power and improves T-maze performance (Mederos et al., 2021); thus astrocyte Gq signaling may have a bidirectional ability to modulate memory. The recently developed ißARK tool attenuates astrocyte Gq-induced calcium activity (Nagai et al., 2021b). Brain-wide expression of i $\beta A R K$ reduces performance in the Y-maze and the modified novel object placement test (Nagai et al., 2021b). Here, contextual fear conditioning and cued memory are unaffected (Nagai et al., 2021b) suggesting effects only on specific types of memory. While differences in tools used, timescales of activation or attenuation, brain region, and behavioral assays employed make direct comparisons difficult, these recent studies indicate that $\mathrm{Gq}$ signaling is indeed critical to astrocyte modulation of memory and hint at regional differences in astrocyte modulation of circuits and behavior.

Fewer studies have investigated the role of astrocyte Gi-GPCR signaling in regulation of memory. Dorsal hippocampal $\mathrm{Gi}$ activation attenuates stress-enhanced fear learning
(Jones et al., 2018), while CA1 astrocyte Gi activation impairs memory recall and inhibits CA1 to anterior cingulate cortex projections important to memory recall, but not recent memory (Kol et al., 2020). Gi-GPCR signaling is the endogenous signaling pathway activated in astrocytes by $\mu$-opioid receptor agonism and $\mathrm{Gi}$ signaling in CA1 astrocytes is implicated in memory acquisition in conditioned place preference (Nam et al., 2019) suggesting the presence of astrocyte subtypes involved in different types of memory within the hippocampus.

Lastly, astrocyte Gs-GPCR signaling affects memory. Typically, Gs stimulates adenylyl cyclase and increases the second messenger cyclic AMP (cAMP) (Neves et al., 2002). In hippocampal and striatal astrocytes, Gs signaling increases calcium signaling though to a lesser extent than $\mathrm{Gq}$ signaling (Chai et al., 2017). Activation of Rs1, a modified Gs-coupled receptor (Table 1), in hippocampal and thalamic astrocytes impairs spatial memory in the Morris water maze when activated during training or one day later (Orr et al., 2015). 
Conversely, elevating hippocampal astrocyte cAMP through a photoactivated adenylyl cyclase (Table 1) during or immediately after training improved performance in a different memory test, the novel object placement test (Zhou et al., 2021). In this same study, increasing cAMP one day after training impaired memory retention (Zhou et al., 2021). Differences in the effect on memory may stem from different memory assays used or different timescales of activation. Orr et al. (2015) report some ligand-independent constitutive Gs activity which reduced novel object recognition, even in the absence of chemogenetic ligand. Long-term Gs activation in hippocampal astrocytes may generally impair memory while acute cAMP increases seem to improve different aspects of memory. Zhou et al. (2021) speculate that increased cAMP during memory formation improves performance through hippocampal neuron long term potentiation but that increased cAMP the following day could reduce the original memory through the same mechanism akin to "re-writing" or replacing the memory. Memory is an energetically demanding process, and in addition to the roles discussed here, astrocytes also influence memory by providing energy to neurons through astrocyte-neuron metabolic coupling (Suzuki et al., 2011; Alberini et al., 2018).

\section{Sensory and Motor}

Astrocytes respond to sensory and motor activity through elevations in intracellular calcium signaling. For example, astrocyte calcium signaling increases in response to the stimulation of whiskers, in a frequency-dependent manner (Wang et al., 2006), noting this study was conducted in anesthetized mice which may have altered astrocyte activity. Advances in two-photon imaging with stable head-mounted microscopes allowed for imaging of astrocyte calcium activity in awake behaving mice (Dombeck et al., 2007). Using two-photon imaging with cellular resolution, Dombeck et al. (2007) found calcium activity correlated with running in a subset of sensory cortex astrocytes in awake behaving mice. Similar techniques revealed increased barrel cortex astrocyte endfoot calcium activity in response to natural hyperemia during whisking or locomotion (Tran et al., 2018), demonstrating astrocyte calcium responses to vascular signaling dependent on behavioral state.

In the cerebellum, large networks of radial astrocytes called Bergmann glia display increased calcium activity at the onset of locomotion (Nimmerjahn et al., 2009). Channelrhodopsin stimulation of Bergmann glia increases the horizontal optokinetic reflex and pupil size, both known to be modulated by cerebellar circuits (Sasaki et al., 2012), suggesting a role for Bergmann glia in visual reflexes. Channelrhodopsin activation of V1 astrocytes altered the visual response properties of local neurons affecting both local parvalbumin and somatostatin interneuron excitability (Perea et al., 2014). Calcium signaling in visual cortex astrocytes shows responsiveness to visual stimuli (Schummers et al., 2008). Thus, astrocyte calcium activity correlates with behavioral outputs and manipulation of astrocyte activity alters sensory behavior.

More recently, astrocyte calcium imaging paired with electrocorticogram monitoring of neuronal activity revealed that somatosensory cortex astrocytes respond to sensory stimuli in a stimulus-dependent manner in anesthetized mice
(Lines et al., 2020). Application of an electrode to the hindpaw induces cortical neuron gamma activity, thought to underlie cognitive processes, followed by increased astrocyte calcium activity that appears to tamp down neuronal gamma activity. This neuronal gamma activity remained high in Itpr 2 knockout mice with blunted astrocyte calcium signaling indicating astrocyte regulation of sensory-evoked neuronal activity. Likewise, astrocyte Gq activation by hM3Dq DREADD reduced sensory-evoked neuronal gamma activity (Lines et al., 2020). DN-SNARE mice also show reduced cortical gamma oscillations (Lee et al., 2014) suggesting a role for gliotransmitters. Lastly, astrocytes may also modulate tactile discrimination. In the ventrobasal nucleus of the thalamus, astrocytes are the reported source of tonic GABA inhibition important to tactile discrimination. Local infusion of GABA reduces tactile acuity while astrocyte-specific knockdown of GABA synthesis pathway genes increased tactile acuity (Kwak et al., 2020). Therefore astrocytes regulate neuronal responses to sensory stimuli both within localized circuits and at the level of cortical neuron networks.

\section{Feeding}

Astrocytes actively regulate metabolic homeostasis and feeding behavior. Changes in diet or fasting alter astrocyte gene expression, morphology, and neuronal ensheathment and astrocytes are responsive to glucose and the hormones insulin, leptin, and ghrelin (Hsuchou et al., 2009; Horvath et al., 2010; Fuente-Martin et al., 2012; Kim et al., 2014; Buckman et al., 2015; Garcia-Caceres et al., 2016, 2019; Varela et al., 2021). Hypothalamic neurons regulate feeding behavior and agoutirelated peptide (AgRP) neurons of the arcuate nucleus (ARC) in the ventral floor of the mediobasal hypothalamus (MBH) drive feeding behavior (Wu and Palmiter, 2011; Andermann and Lowell, 2017). However, chemogenetic manipulation of MBH astrocytes has yielded conflicting results. In one study, hM3Dq DREADD activation of MBH astrocytes reduced basal food intake as well as feeding induced by the orexigenic hormone ghrelin (Yang et al., 2015). Conversely, Chen et al. (2016) found hM3Dq DREADD activation of ARC astrocytes increased food intake. As AgRP neuron activation is known to increase feeding behavior (Aponte et al., 2011; Krashes et al., 2011), both studies queried the effect of astrocyte Gq-activation on AgRP neuron activity. Yang et al. (2015) reported astrocyte inhibition of AgRP neurons via adenosine while Chen et al. (2016) reported facilitation of AgRP neurons by ARC astrocytes. In the latter study, astrocyte-mediated increase in food intake was dependent upon AgRP neuron activity as hM4Di inhibition of AgRP neurons alongside astrocyte activation did not increase food intake. These discrepancies could stem from targeting of the entire MBH (Yang et al., 2015) versus specifically the ARC (Chen et al., 2016) and may indicate region-specific roles for astrocytes in modulation of food intake.

Interestingly, Gi-coupled hM4Di signaling in MBH astrocytes increased and prolonged ghrelin-evoked feeding but did not affect basal feeding (Yang et al., 2015) noting that hM4Di signaling has more recently been shown to increase astrocyte calcium signaling and promote gliotransmitter release (Chai et al., 2017; Durkee et al., 2019), in contrast to inhibition 
observed in neurons. Reducing astrocyte calcium signaling through buffering of IP3 by p130PH (Xie et al., 2010; Table 1) reduced food intake (Chen et al., 2016). Chen et al. (2016) saw no difference in food intake with hM4Di signaling suggesting that different GPCR signaling pathways within hypothalamic astrocytes may have different behavioral effects, as in the cortex (Vaidyanathan et al., 2021).

The above discrepancies may also arise from food intake analyses across different phases of the light-dark cycle or differing CNO dosage ( $5 \mathrm{mg} / \mathrm{kg}$ vs. $0.3 \mathrm{mg} / \mathrm{kg}$ ). It is important to note that both studies used the GFAP promoter to target DREADD expression to hypothalamic astrocytes which also targets tanycytes lining the third ventricle, that are also implicated in feeding and energy homeostasis (Bolborea and Dale, 2013; Chen et al., 2016). An additional caveat is neither study assayed additional behaviors such as locomotor or anxiety-like behavior, which may affect feeding behavior and are also modulated by AgRP neurons (Dietrich et al., 2015; Padilla et al., 2016). Optogenetic activation of $\mathrm{MBH}$ astrocytes reduced food intake as well as fasting- or ghrelin-induced increases in food intake, also in an A1-receptor dependent manner (Sweeney et al., 2016). Here, locomotor or anxiety-like behavior were assayed but were not affected by optogenetic stimulation of $\mathrm{MBH}$ astrocytes.

While the differing roles of ARC versus all $\mathrm{MBH}$ astrocytes in feeding and their effects on AgRP neurons remain to be further elucidated, recent research corroborates a role for ARC astrocytes in facilitation of AgRP neuron excitement (Varela et al., 2021). AgRP neuron release of GABA promotes increased glial ensheathment of AgRP neurons resulting in increased AgRP neuron excitability and thus a feed-forward loop driving AgRP neuron excitability (Varela et al., 2021). While this work does not directly test behavior, increases in AgRP neuron excitability are expected to increase feeding behavior (Aponte et al., 2011; Krashes et al., 2011). Astrocytes are additionally implicated in satiety signals. Astrocytes in the dorsal vagal complex are activated after 12 hours of feeding on a high fat diet. Chemogenetic activation of these astrocytes via $\mathrm{hM} 3 \mathrm{Dq}$ expression reduced basal and post-fasting food intake (MacDonald et al., 2020). Future in vivo studies may employ simultaneous monitoring of neuronal and astrocyte calcium signaling during feeding behavior, alongside astrocyte manipulations, to further resolve the role of astrocytes in feeding in a region-specific manner.

\section{Fear and Anxiety}

Both the hippocampus and the amygdala are important for fear and anxiety (Davis, 1992; Maren, 2008). As discussed in the Memory section, increased Gq signaling in hippocampal astrocytes promoted freezing in the contextual fear conditioning assay (Adamsky et al., 2018) while brain-wide reduction of Gq signaling did not alter freezing (Nagai et al., 2021b). Gi-GPCR activation in the dorsal hippocampus attenuated stress-enhanced fear learning (Jones et al., 2018). In the amygdala, astrocytes modulate fear responses through regulation of synapses in the medial subdivision of the central amygdala (CeM) (MartinFernandez et al., 2017). Gq DREADD activation of CeM astrocytes reduced local neuron firing rate and decreased fear expression while anxiety-like behavior, as measured by the elevated plus maze, was unaffected (Martin-Fernandez et al., 2017). Knockdown of astrocyte glucocorticoid receptors in the central amygdala attenuates fear memory and reduces anxietylike behavior in the open field test (Wiktorowska et al., 2021). Basolateral amygdala astrocytes are also implicated in the modulation of fear extinction, and chemogenetic Gq-GPCR activation facilitates fear extinction (Shelkar et al., 2021). Future studies are required to further delineate the role of astrocytes in fear and anxiety though current evidence indicates amygdala astrocyte activity may reduce fear behavior, while hippocampal astrocyte activity has variable effects, potentially dependent on different GPCR signaling pathways or region. A subset of astrocytes in the lateral and capsular part of the central amygdala (CeL) express oxytocin receptors (OTR) and respond to oxytocin signaling with increased calcium signaling that propagates to neighboring astrocytes via gap junctions (Wahis et al., 2021a). Downstream neuronal NMDA receptor activation increases CeL interneuron excitability ultimately increasing inhibitory input onto medial central amygdala projection neurons. Behaviorally, OTR signaling in these astrocytes has an anxiolytic effect both in pain-free conditions and in a model of neuropathic pain (Wahis et al., 2021a) indicating a role for OTR-expressing astrocytes of the amygdala in anxiety. Future studies are required to understand how other astrocyte subtypes of the CeL influence behavior and, more generally, how interactions of different astrocyte subtypes affect local circuity and downstream behavior.

\section{Cognitive Processes}

Understanding of neuronal regulation of cognitive functions like learning, memory, attention, and decision-making has greatly advanced in the last two decades (Roth and Ding, 2020) yet the role of astrocytes in cognitive processes remains relatively unknown. Memory (discussed above) is the most well-studied, and in this last section we will discuss a series of recent studies demonstrating astrocyte contributions to other aspects of cognitive functioning.

Behavioral flexibility is critical to achieving a goal as it allows an organism to pivot to an alternative strategy or to stop the current behavior and preserve energy after repeated failures. In zebrafish, radial astrocytes modulate these adaptative behavioral responses. With increasing failed attempts at swimming, noradrenergic neurons signal to astrocytes which accumulate this "failure signal" as increasing intracellular calcium. Astrocytes then activate GABA neurons that drive cessation of swimming (Mu et al., 2019). In mice, noradrenaline is also implicated in "priming" astrocytes to respond to changes in cortical neuron network activity (Paukert et al., 2014) indicating that astrocyte networks respond to behavioral state.

In the striatum, different astrocyte populations differentially regulate goal-directed behavior. Habitual behavior can become detrimental when the reward or goal has changed. Chemogenetic activation of dorsomedial striatum astrocyte $\mathrm{Gq}$ signaling promotes flexible goal-directed behavior in an operant paradigm in which control mice without astrocyte activation continued to demonstrate habitual behavior (Kang et al., 2020). Astrocyte $\mathrm{Gq}$ activation differentially affected the activity of local indirect 
and direct pathway medium spiny neurons (MSNs). In this same study, mice lacking ENT1, an astrocyte adenosine transporter, did not transition from habitual to goal-directed behavior indicating a role for astrocyte adenosine in behavioral flexibility. Astrocyte regulation of extracellular glutamate levels, by the glutamate transporter EAAT2 (alias GLT-1), is also implicated in behavioral flexibility (Boender et al., 2021). EAAT2 expression rises in dorsolateral striatal astrocytes with increased habitual behavioral inflexibility in an operant paradigm. Yet, chemogenetic activation of astrocyte Gq signaling during overtraining in the operant paradigm reduces EAAT2 expression and restores behavioral flexibility. Thus, neuron-astrocyte interactions underly aspects of behavioral flexibility.

Striatal astrocytes are also implicated in attention. Chemogenetic activation of astrocyte Gi-GPCR pathways, mimicking endogenous $\mathrm{GABA}_{B}$ agonism, increases excitatory synapse number, MSN firing rate, and hyperactivity behavior in the open field assay. Further, attention is disrupted in both a modified open field assay and novel object recognition test (Nagai et al., 2019). These synaptic and behavioral effects were reversible and returned to control levels 48 hours post-CNO injection. Astrocyte Gi activation through hM4Di DREADD also increased astrocyte thrombospondin-1 and blockade of the thrombospondin-1 receptor rescues synaptic and behavioral deficits (Nagai et al., 2019). The authors propose a model in which MSN-released GABA induces astrocyte release of the synaptogenic cue thrombospondin1 , inducing synapse formation and increasing excitatory transmission from MSNs resulting in behavioral alterations. These findings suggest that astrocyte secreted thrombospondin1 can affect behavior over a short timescale, highlighting the ability of astrocyte secreted factors to affect behavior, either independently of, or alongside, gliotransmission (Nagai et al., 2019). Striatal astrocytes are further implicated in repetitive self-grooming behavior. Reduction of striatal astrocyte calcium signaling through overexpression of a modified calcium plasma membrane pump (CalEx) altered local MSN activity and increased self-grooming without affecting motor ability or anxiety-like behavior (Yu et al., 2018).

Lastly, astrocytes are implicated in reward and addiction behavior. Studies have employed astrocyte Gq-GPCR activation to query the role of astrocytes in ethanol intake. Activation of PFC astrocytes increased ethanol consumption in ethanolnaïve male mice whereas reduction of astrocyte calcium via CalEx expression reduced ethanol drinking indicating a bidirectional regulation of ethanol-consumption by PFC astrocytes (Erickson et al., 2021). In the basolateral amygdala, activation of astrocytes reduced ethanol consumption in male mice (Nwachukwu et al., 2021). Likewise, in the nucleus accumbens (NAc), a key reward center, activation of astrocytes in male rats decreased ethanol-seeking behavior after abstinence (Bull et al., 2014). Further, activation of NAc astrocytes in female mice reduced reward-seeking behavior in ethanol dependent mice (Giacometti et al., 2020). Ethanol exposure alters gene expression in astrocytes in a sex-specific manner (Wilhelm et al., 2016) however, none of the above studies tested both male and female mice making direct comparisons of behavior difficult. Future studies are required to identify if astrocytes contribute to sex-specific ethanol-use and reward behavior.

NAc Gq-GPCR activation in male rats also reduced cueinduced reinstatement of cocaine seeking but did not affect initial cocaine self-administration indicating a potential role for astrocytes in relapse behavior (Scofield et al., 2015). Generally, NAc astrocyte activity correlates with reduced substance seeking and reduced relapse. Recently, Corkrum et al. (2020) demonstrated that NAc astrocytes respond to dopamine with increased calcium signaling, a response augmented by amphetamine, and absent in Itpr2 knockout mice or in the presence of $\mathrm{G}$ protein signaling blockade. Chemogenetic GqGPCR activation of NAc astrocytes depresses local excitatory neuronal transmission, via adenosine A1 receptor signaling. Further, Itpr2 knockout mice and mice lacking dopamine D1 receptors in astrocytes showed reduced amphetamineinduced locomotor activity (Corkrum et al., 2020) indicating a role for astrocytes in the behavioral effects of amphetamine and dopaminergic circuitry. Therefore NAc astrocytes regulate reward and addiction behavior, in part, through dopamine responsivity. Future studies may examine astrocytes as a therapeutic target for addiction treatment.

\section{CHALLENGES AND FUTURE PROSPECTS}

In this review we have discussed the role of astrocytes in regulation of behavior. Acute manipulation of astrocytes with chemogenetic and optogenetic tools allow for the probing of causal relationships between astrocyte activity and animal behavior. This is in contrast to earlier gene knockout, or even inducible knockout studies, that relied on the manipulation of astrocytes over a timescale that might allow for compensatory mechanisms to affect circuitry, neuronal networks, and downstream behavior. Indeed, the ability of astrocytes to regulate synapses extends to larger circuitry and behavioral outputs. Future studies of neural circuits and behavior must therefore consider astrocyte contributions and may even incorporate astrocytes into their circuit diagrams or computational models. While not discussed here, it will also be important to consider the impact of other brain cells such as microglia (Yirmiya and Goshen, 2011; Werneburg et al., 2017), oligodendrocytes (Suminaite et al., 2019; Xin and Chan, 2020), and vascular cells (Filosa et al., 2016; Stackhouse and Mishra, 2021) on neuronal and astrocyte activity, and ultimately behavior.

Toward further understanding how astrocyte gliotransmission affects local circuitry and behavior in vivo, there is a growing list of neurochemical sensors that may provide insight (Looger and Griesbeck, 2012; Scofield et al., 2015; Beyene et al., 2019; Leopold et al., 2019). Many studies reviewed here focused on astrocyte interactions with local neuronal circuitry, as would be expected given that astrocytes do not project to other brain regions as neurons do. However, future studies, and the tools used for experiments, must consider the syncytium of astrocytes connected via astrocyte gap junctions (Landis and Reese, 1982) and the potential spreading of any manipulation within this 
network. Further, astrocyte gene expression changes across development and into adulthood (Boisvert et al., 2018) therefore it remains critical to consider developmental timing in choice of tool for behavioral studies.

\section{Expanding the Genetic Toolbox for Probing Astrocyte Heterogeneity and Function}

The currently used Aldh1l1 (Tsai et al., 2012), GFAP (Lee et al., 2008), and GfaABC1D (Shigetomi et al., 2013; Figure 2) promoters efficiently target astrocytes but, in some cases, may target non-astrocyte cells such as the tanycytes lining the ventricle (Chen et al., 2016) or subsets of neurons (Cahoy et al., 2008; Farhy-Tselnicker et al., 2017). Numerous recent papers highlight the transcriptional diversity of astrocytes (Cahoy et al., 2008; Zhang et al., 2014; Srinivasan et al., 2016; Chai et al., 2017; Boisvert et al., 2018; Clarke et al., 2018; Batiuk et al., 2020; Bayraktar et al., 2020; Farhy-Tselnicker et al., 2020; Guttenplan et al., 2020; Pan et al., 2020). These databases of astrocyte gene expression may identify new astrocyte specific genetic markers for astrocyte targeting. Further, they may allow for the functional interrogation of astrocyte subtypes, either through genetic markers specific to astrocyte subtypes or through intersectional genetics using existing astrocyte Cre-dependent tools paired with new subtype-specific Flp-dependent tools (Figure 2B; Dymecki et al., 2010; Fenno et al., 2014). Already there is evidence for a small population of genetically distinct astrocytes serving an important behavioral role. The OTRexpressing subtype of central amygdala astrocytes makes up around $19 \%$ of all astrocytes in that brain region yet mediates the anxiolytic effects of oxytocin (Wahis et al., 2021a). Future experiments should address the behavioral role of the remaining central amygdala astrocytes as well as exploring astrocyte subtype influences on behavior in other brain regions. If a local astrocyte subtype mediates one behavioral function, might a nearby subtype mediate an opposing behavioral function? Following identification of functional astrocyte subtypes, the next step will be to probe their interactions during naturalistic behaviors. Such experiments will be technically challenging and will likely require two photon imaging with multicolor calcium indicators paired with advanced ethologically relevant behavior tracking (Mathis et al., 2018; Wiltschko et al., 2020). As noradrenaline exerts behavioral state-specific effects on large populations of astrocytes (Paukert et al., 2014), an additional challenge will be to understand how more global astrocyte activity resulting from neuromodulation interacts with local circuit- or subtype-specific astrocyte activity (Bazargani and Attwell, 2016).

\section{Increasing Understanding of Astrocyte Endogenous Signaling Mechanisms}

Many of the tools discussed here target astrocyte GPCRs ultimately leading to increased calcium signaling ( $\mathrm{Yu}$ et al., 2020). Key questions toward resolving astrocyte contributions to behavioral regulation include, (1) what additional intracellular signaling pathways are involved, (2) how is astrocyte gene expression affected, and (3) how does spatially localized astrocyte calcium activity influence behavior? Signaling pathways following GPCR activation in astrocytes are not fully resolved and should be the focus of future investigations. Understanding these molecular mechanisms will also inform tool design for physiologically relevant methods to manipulate astrocytes and probe involvement in behavioral regulation. Future studies should also address how GPCR signaling in astrocytes may alter gene expression. RNA sequencing following GPCR activation can reveal changes in gene expression that may underlie astrocyte regulation of synapses, circuits, and behavior. For example, how Gi-GPCR activation of striatal astrocytes mechanistically increases hyperactivity and attention was revealed through RNA sequencing of astrocytes following acute Gi-GPCR activation compared to control animals, revealing higher expression of the synaptogenic cue thrombospondin-1 (Nagai et al., 2019). As astrocyte processes display spontaneous and spatially localized calcium transients that can occur independently of calcium release from the endoplasmic reticulum (Shigetomi et al., 2010; Di Castro et al., 2011; Khakh and McCarthy, 2015; Bazargani and Attwell, 2016; Rungta et al., 2016; Agarwal et al., 2017), future studies should probe the involvement of spatially localized astrocyte calcium activity in behavior. For example, SpiCee is a recently developed genetically encoded calcium chelator designed to manipulate intracellular calcium levels within different subcellular regions (Ros et al., 2020) which may prove useful for such studies. Research on astrocyte intracellular signaling pathways, gene expression, and calcium localization will continue to inform on how astrocytes shape behavior.

\section{Sex Differences in Astrocytes and Behavior}

Neuroscience research in mice has regularly focused on male subjects with one analysis finding only $20 \%$ of studies used both male and female mice while around $25 \%$ did not state the sex of subjects (Beery and Zucker, 2011; Prendergast et al., 2014). Cortical astrocytes show significant sex differences in gene expression during development and astrocytes express estrogen receptors (Wright et al., 2010; Rurak et al., 2020). As noted above, ethanol consumption has sex-specific effects on gene expression in astrocytes (Wilhelm et al., 2016) indicating the potential for environmental factors to effect astrocyte function in sex-specific ways. Recently, astrocytederived thrombospondin-1 was reported to induce cortical synaptogenesis in a sex-specific manner (Mazur et al., 2021). Taken together, sex differences in astrocyte regulation of behavior are likely but remain unexplored. Future studies should employ both male and female mice to further elucidate sex differences in astrocytes. As astrocytes also contribute to pathological behavior in disordered states (Dallerac and Rouach, 2016; Santello et al., 2019), the use of male and female mice in astrocyte research will have important implications for human health (Beery and Zucker, 2011).

\section{CONCLUSION}

Research reviewed here demonstrates the importance of astrocytes in the regulation of homeostatic functions and diverse behavioral processes. Astrocytes can influence local neuronal 
circuitry through release of secreted factors or gliotransmitters leading to changes in synaptic structure and altered neuronal excitability. Through these capacities, astrocytes are able to shape behavior via changes in neuronal activity (Figure 3). In turn, neuronal activity can influence astrocyte activity thus these interactions are bidirectional. The majority of studies reviewed here parsed astrocyte behavioral function in circuits known to modulate a specific behavioral function. Acute manipulation of hypothalamic astrocytes influences feeding (Yang et al., 2015; Chen et al., 2016), hippocampal astrocytes impact memory (Adamsky et al., 2018; Mederos et al., 2019, 2021; Kol et al., 2020), and amygdala astrocytes regulate fear (Martin-Fernandez et al., 2017). These functions are already known to be subserved by those brain regions and now astrocyte contributions are beginning to be appreciated. Future studies may reveal surprising roles for astrocytes in behavioral regulation, independent of known neuronal circuit functions. As understanding of astrocyte heterogeneity increases, future experiments will address whether astrocytes perform subtypespecific functions underlying neuronal circuit activity and organismal behavior.

\section{REFERENCES}

Abel, T., and Klann, E. (2013). Molecular and cellular cognition: neurobiology of learning and memory special issue 2013. Neurobiol. Learn. Mem. 105, 1-2. doi: 10.1016/j.nlm.2013.08.005

Adamsky, A., Kol, A., Kreisel, T., Doron, A., Ozeri-Engelhard, N., Melcer, T., et al. (2018). Astrocytic activation generates de novo neuronal potentiation and memory enhancement. Cell 174, 59.e14-71.e14. doi: 10.1016/j.cell.2018.05.002

Agarwal, A., Wu, P. H., Hughes, E. G., Fukaya, M., Tischfield, M. A., Langseth, A. J., et al. (2017). Transient opening of the mitochondrial permeability transition pore induces microdomain calcium transients in astrocyte processes. Neuron 93, 587.e587-605.e587. doi: 10.1016/j.neuron.2016.12.034

Agulhon, C., Boyt, K. M., Xie, A. X., Friocourt, F., Roth, B. L., and McCarthy, K. D. (2013). Modulation of the autonomic nervous system and behaviour by acute glial cell Gq protein-coupled receptor activation in vivo. J. Physiol. 591, 5599-5609. doi: 10.1113/jphysiol.2013.261289

Agulhon, C., Petravicz, J., McMullen, A. B., Sweger, E. J., Minton, S. K., Taves, S. R., et al. (2008). What is the role of astrocyte calcium in neurophysiology? Neuron 59, 932-946. doi: 10.1016/j.neuron.2008.09.004

Alberini, C. M., Cruz, E., Descalzi, G., Bessieres, B., and Gao, V. (2018). Astrocyte glycogen and lactate: new insights into learning and memory mechanisms. Glia 66, 1244-1262. doi: 10.1002/glia.23250

Alexander, G. M., Rogan, S. C., Abbas, A. I., Armbruster, B. N., Pei, Y., Allen, J. A., et al. (2009). Remote control of neuronal activity in transgenic mice expressing evolved G protein-coupled receptors. Neuron 63, 27-39. doi: 10.1016/j.neuron. 2009.06.014

Allen, N. J., and Barres, B. A. (2009). Neuroscience: glia - more than just brain glue. Nature 457, 675-677. doi: 10.1038/457675a

Andermann, M. L., and Lowell, B. B. (2017). Toward a wiring diagram understanding of appetite control. Neuron 95, 757-778. doi: 10.1016/j.neuron. 2017.06.014

Aponte, Y., Atasoy, D., and Sternson, S. M. (2011). AGRP neurons are sufficient to orchestrate feeding behavior rapidly and without training. Nat. Neurosci. 14, 351-355. doi: 10.1038/nn.2739

Araque, A., Carmignoto, G., Haydon, P. G., Oliet, S. H., Robitaille, R., and Volterra, A. (2014). Gliotransmitters travel in time and space. Neuron 81, 728-739. doi: 10.1016/j.neuron.2014.02.007

Araque, A., Sanzgiri, R. P., Parpura, V., and Haydon, P. G. (1999). Astrocyteinduced modulation of synaptic transmission. Can. J. Physiol. Pharmacol. 77, 699-706. doi: 10.1139/y99-076

\section{AUTHOR CONTRIBUTIONS}

Both authors conceptualized and wrote this review together. Both authors contributed to the article and approved the submitted version.

\section{FUNDING}

NA was supported by the National Institutes of Health (R01 NS105742, R01 NS089791, and RF1 DA048816) and the Chan Zuckerberg Initiative. KL was supported by the National Institute of Neurological Disorders and Stroke of the National Institutes of Health (K00 NS108515).

\section{ACKNOWLEDGMENTS}

We thank Adrien Paumier, Laura Sancho, Kathryn Lehigh, and Ashley Brandebura for comments on the manuscript. Figures were created using BioRender.

Asrican, B., Wooten, J., Li, Y. D., Quintanilla, L., Zhang, F., Wander, C., et al. (2020). Neuropeptides modulate local astrocytes to regulate adult hippocampal neural stem cells. Neuron 108, 349.e346-366.e346. doi: 10.1016/j.neuron.2020. 07.039

Bang, J., Kim, H. Y., and Lee, H. (2016). Optogenetic and chemogenetic approaches for studying astrocytes and gliotransmitters. Exp. Neurobiol. 25, 205-221. doi: 10.5607/en.2016.25.5.205

Barnes, C. A. (1979). Memory deficits associated with senescence: a neurophysiological and behavioral study in the rat. J. Comp. Physiol. Psychol. 93, 74-104. doi: 10.1037/h0077579

Batiuk, M. Y., Martirosyan, A., Wahis, J., de Vin, F., Marneffe, C., Kusserow, C., et al. (2020). Identification of region-specific astrocyte subtypes at single cell resolution. Nat. Commun. 11:1220. doi: 10.1038/s41467-019-14198-8

Bayraktar, O. A., Bartels, T., Holmqvist, S., Kleshchevnikov, V., Martirosyan, A., Polioudakis, D., et al. (2020). Astrocyte layers in the mammalian cerebral cortex revealed by a single-cell in situ transcriptomic map. Nat. Neurosci. 23, 500-509. doi: 10.1038/s41593-020-0602-1

Bazargani, N., and Attwell, D. (2016). Astrocyte calcium signaling: the third wave. Nat. Neurosci. 19, 182-189. doi: 10.1038/nn.4201

Beery, A. K., and Zucker, I. (2011). Sex bias in neuroscience and biomedical research. Neurosci. Biobehav. Rev. 35, 565-572. doi: 10.1016/j.neubiorev.2010. 07.002

Ben Achour, S., and Pascual, O. (2012). Astrocyte-neuron communication: functional consequences. Neurochem. Res. 37, 2464-2473. doi: 10.1007/s11064012-0807-0

Beyene, A. G., Yang, S. J., and Landry, M. P. (2019). Review article: tools and trends for probing brain neurochemistry. J. Vac. Sci. Technol. A 37:040802. doi: 10.1116/1.5051047

Bezzi, P., Gundersen, V., Galbete, J. L., Seifert, G., Steinhauser, C., Pilati, E., et al. (2004). Astrocytes contain a vesicular compartment that is competent for regulated exocytosis of glutamate. Nat. Neurosci. 7, 613-620. doi: 10.1038/ nn 1246

Bezzi, P., and Volterra, A. (2001). A neuron-glia signalling network in the active brain. Curr. Opin. Neurobiol. 11, 387-394. doi: 10.1016/s0959-4388(00)0 0223-3

Blum, I. D., Keles, M. F., Baz, E. S., Han, E., Park, K., Luu, S., et al. (2021). Astroglial calcium signaling encodes sleep need in drosophila. Curr. Biol. 31, 150.e157-162.e157. doi: 10.1016/j.cub.2020.10.012

Boender, A. J., Bontempi, L., Nava, L., Pelloux, Y., and Tonini, R. (2021). Striatal astrocytes shape behavioral flexibility via regulation of the glutamate 
transporter EAAT2. Biol. Psychiatry 89, 1045-1057. doi: 10.1016/j.biopsych. 2020.11.015

Boisvert, M. M., Erikson, G. A., Shokhirev, M. N., and Allen, N. J. (2018). The aging astrocyte transcriptome from multiple regions of the mouse brain. Cell Rep. 22, 269-285. doi: 10.1016/j.celrep.2017.12.039

Bojarskaite, L., Bjornstad, D. M., Pettersen, K. H., Cunen, C., Hermansen, G. H., Abjorsbraten, K. S., et al. (2020). Astrocytic $\mathrm{Ca}(2+)$ signaling is reduced during sleep and is involved in the regulation of slow wave sleep. Nat. Commun. 11:3240. doi: 10.1038/s41467-020-17062-2

Bolborea, M., and Dale, N. (2013). Hypothalamic tanycytes: potential roles in the control of feeding and energy balance. Trends Neurosci. 36, 91-100. doi: 10.1016/j.tins.2012.12.008

Boyden, E. S., Zhang, F., Bamberg, E., Nagel, G., and Deisseroth, K. (2005). Millisecond-timescale, genetically targeted optical control of neural activity. Nat. Neurosci. 8, 1263-1268. doi: 10.1038/nn1525

Brancaccio, M., Edwards, M. D., Patton, A. P., Smyllie, N. J., Chesham, J. E., Maywood, E. S., et al. (2019). Cell-autonomous clock of astrocytes drives circadian behavior in mammals. Science 363, 187-192. doi: 10.1126/science. aat 4104

Brancaccio, M., Patton, A. P., Chesham, J. E., Maywood, E. S., and Hastings, M. H. (2017). Astrocytes control circadian timekeeping in the suprachiasmatic nucleus via glutamatergic signaling. Neuron 93, 1420.e1425-1435.e1425. doi: 10.1016/j.neuron.2017.02.030

Buckman, L. B., Thompson, M. M., Lippert, R. N., Blackwell, T. S., Yull, F. E., and Ellacott, K. L. (2015). Evidence for a novel functional role of astrocytes in the acute homeostatic response to high-fat diet intake in mice. Mol. Metab. 4, 58-63. doi: 10.1016/j.molmet.2014.10.001

Bull, C., Freitas, K. C., Zou, S., Poland, R. S., Syed, W. A., Urban, D. J., et al. (2014). Rat nucleus accumbens core astrocytes modulate reward and the motivation to self-administer ethanol after abstinence. Neuropsychopharmacology 39, 28352845. doi: 10.1038/npp.2014.135

Cahoy, J. D., Emery, B., Kaushal, A., Foo, L. C., Zamanian, J. L., Christopherson, K. S., et al. (2008). A transcriptome database for astrocytes, neurons, and oligodendrocytes: a new resource for understanding brain development and function. J. Neurosci. 28, 264-278. doi: 10.1523/JNEUROSCI.4178-07.2008

Chai, H., Diaz-Castro, B., Shigetomi, E., Monte, E., Octeau, J. C., Yu, X., et al. (2017). Neural circuit-specialized astrocytes: transcriptomic, proteomic, morphological, and functional evidence. Neuron 95, 531.e539-549.e539. doi: 10.1016/j.neuron.2017.06.029

Chen, N., Sugihara, H., Kim, J., Fu, Z., Barak, B., Sur, M., et al. (2016). Direct modulation of GFAP-expressing glia in the arcuate nucleus bi-directionally regulates feeding. Elife 5:e18716. doi: 10.7554/eLife.18716

Clarke, L. E., Liddelow, S. A., Chakraborty, C., Munch, A. E., Heiman, M., and Barres, B. A. (2018). Normal aging induces A1-like astrocyte reactivity. Proc. Natl. Acad. Sci. U.S.A. 115, E1896-E1905. doi: 10.1073/pnas.1800165115

Clasadonte, J., Scemes, E., Wang, Z., Boison, D., and Haydon, P. G. (2017). Connexin 43-mediated astroglial metabolic networks contribute to the regulation of the sleep-wake cycle. Neuron 95, 1365.e1365-1380.e1365. doi: 10.1016/j.neuron.2017.08.022

Corkrum, M., Covelo, A., Lines, J., Bellocchio, L., Pisansky, M., Loke, K., et al. (2020). Dopamine-evoked synaptic regulation in the nucleus accumbens requires astrocyte activity. Neuron 105, 1036.e1035-1047.e1035. doi: 10.1016/j. neuron.2019.12.026

Cornell-Bell, A. H., Finkbeiner, S. M., Cooper, M. S., and Smith, S. J. (1990). Glutamate induces calcium waves in cultured astrocytes: long-range glial signaling. Science 247, 470-473. doi: 10.1126/science.1967852

Dallerac, G., and Rouach, N. (2016). Astrocytes as new targets to improve cognitive functions. Prog. Neurobiol. 144, 48-67. doi: 10.1016/j.pneurobio.2016.01.003

Davis, M. (1992). The role of the amygdala in fear and anxiety. Annu. Rev. Neurosci. 15, 353-375. doi: 10.1146/annurev.ne.15.030192.002033

De Pitta, M., Brunel, N., and Volterra, A. (2016). Astrocytes: orchestrating synaptic plasticity? Neuroscience 323, 43-61. doi: 10.1016/j.neuroscience.2015.04.001

Di Castro, M. A., Chuquet, J., Liaudet, N., Bhaukaurally, K., Santello, M., Bouvier, D., et al. (2011). Local Ca2+ detection and modulation of synaptic release by astrocytes. Nat. Neurosci. 14, 1276-1284. doi: 10.1038/nn.2929

Dietrich, M. O., Zimmer, M. R., Bober, J., and Horvath, T. L. (2015). Hypothalamic Agrp neurons drive stereotypic behaviors beyond feeding. Cell 160, 1222-1232. doi: 10.1016/j.cell.2015.02.024
Dombeck, D. A., Khabbaz, A. N., Collman, F., Adelman, T. L., and Tank, D. W. (2007). Imaging large-scale neural activity with cellular resolution in awake, mobile mice. Neuron 56, 43-57. doi: 10.1016/j.neuron.2007.08.003

Durkee, C. A., and Araque, A. (2019). Diversity and specificity of astrocyte-neuron communication. Neuroscience 396, 73-78. doi: 10.1016/j.neuroscience.2018.11. 010

Durkee, C. A., Covelo, A., Lines, J., Kofuji, P., Aguilar, J., and Araque, A. (2019). Gi/o protein-coupled receptors inhibit neurons but activate astrocytes and stimulate gliotransmission. Glia 67, 1076-1093. doi: 10.1002/glia.23589

Dymecki, S. M., Ray, R. S., and Kim, J. C. (2010). Mapping cell fate and function using recombinase-based intersectional strategies. Methods Enzymol. 477, 183213. doi: 10.1016/S0076-6879(10)77011-7

Erickson, E. K., Dacosta, A. J., Mason, S. C., Blednov, Y. A., Mayfield, R. D., and Harris, R. A. (2021). Cortical astrocytes regulate ethanol consumption and intoxication in mice. Neuropsychopharmacology 46, 500-508. doi: 10.1038/ s41386-020-0721-0

Farhy-Tselnicker, I., and Allen, N. J. (2018). Astrocytes, neurons, synapses: a tripartite view on cortical circuit development. Neural. Dev. 13:7. doi: 10.1186/ s13064-018-0104-y

Farhy-Tselnicker, I., Boisvert, M. M., Liu, H., Dowling, C., Erikson, G. A., BlancoSuarez, E., et al. (2020). Activity-dependent modulation of synapse-regulating genes in astrocytes. bioRxiv [preprint]. doi: 10.1101/2020.12.30.424365

Farhy-Tselnicker, I., van Casteren, A. C. M., Lee, A., Chang, V. T., Aricescu, A. R., and Allen, N. J. (2017). Astrocyte-secreted glypican 4 regulates release of neuronal pentraxin 1 from axons to induce functional synapse formation. Neuron 96, 428.e413-445.e413. doi: 10.1016/j.neuron.2017.09.053

Fenno, L. E., Mattis, J., Ramakrishnan, C., Hyun, M., Lee, S. Y., He, M., et al. (2014). Targeting cells with single vectors using multiple-feature Boolean logic. Nat. Methods 11, 763-772. doi: 10.1038/nmeth.2996

Fiacco, T. A., and McCarthy, K. D. (2004). Intracellular astrocyte calcium waves in situ increase the frequency of spontaneous AMPA receptor currents in CA1 pyramidal neurons. J. Neurosci. 24, 722-732. doi: 10.1523/JNEUROSCI.285903.2004

Figueiredo, M., Lane, S., Stout, R. F. Jr., Liu, B., Parpura, V., Teschemacher, A. G., et al. (2014). Comparative analysis of optogenetic actuators in cultured astrocytes. Cell Calcium 56, 208-214. doi: 10.1016/j.ceca.2014.07.007

Filosa, J. A., Morrison, H. W., Iddings, J. A., Du, W., and Kim, K. J. (2016). Beyond neurovascular coupling, role of astrocytes in the regulation of vascular tone. Neuroscience 323, 96-109. doi: 10.1016/j.neuroscience.2015.03.064

Florian, C., Vecsey, C. G., Halassa, M. M., Haydon, P. G., and Abel, T. (2011). Astrocyte-derived adenosine and A1 receptor activity contribute to sleep lossinduced deficits in hippocampal synaptic plasticity and memory in mice. J. Neurosci. 31, 6956-6962. doi: 10.1523/JNEUROSCI.5761-10.2011

Foley, J., Blutstein, T., Lee, S., Erneux, C., Halassa, M. M., and Haydon, P. (2017). Astrocytic IP3/Ca(2+) signaling modulates theta rhythm and REM sleep. Front. Neural. Circuits 11:3. doi: 10.3389/fncir.2017.00003

Fuente-Martin, E., Garcia-Caceres, C., Granado, M., de Ceballos, M. L., SanchezGarrido, M. A., Sarman, B., et al. (2012). Leptin regulates glutamate and glucose transporters in hypothalamic astrocytes. J. Clin. Invest. 122, 3900-3913. doi: 10.1172/JCI64102

Fujita, T., Chen, M. J., Li, B., Smith, N. A., Peng, W., Sun, W., et al. (2014). Neuronal transgene expression in dominant-negative SNARE mice. J. Neurosci. 34, 16594-16604. doi: 10.1523/JNEUROSCI.2585-14.2014

Garcia-Caceres, C., Balland, E., Prevot, V., Luquet, S., Woods, S. C., Koch, M., et al. (2019). Role of astrocytes, microglia, and tanycytes in brain control of systemic metabolism. Nat. Neurosci. 22, 7-14. doi: 10.1038/s41593-018-0286-y

Garcia-Caceres, C., Quarta, C., Varela, L., Gao, Y., Gruber, T., Legutko, B., et al. (2016). Astrocytic insulin signaling couples brain glucose uptake with nutrient availability. Cell 166, 867-880. doi: 10.1016/j.cell.2016.07.028

Garcia-Marin, V., Garcia-Lopez, P., and Freire, M. (2007). Cajal's contributions to glia research. Trends Neurosci. 30, 479-487. doi: 10.1016/j.tins.2007. 06.008

Giacometti, L. L., Chandran, K., Figueroa, L. A., and Barker, J. M. (2020). Astrocyte modulation of extinction impairments in ethanol-dependent female mice. Neuropharmacology 179:108272. doi: 10.1016/j.neuropharm.2020.108272

Gourine, A. V., Kasymov, V., Marina, N., Tang, F., Figueiredo, M. F., Lane, S., et al. (2010). Astrocytes control breathing through $\mathrm{pH}$-Dependent release of ATP. Science 329, 571-575. doi: 10.1126/science.1190721 
Guerra-Gomes, S., Sousa, N., Pinto, L., and Oliveira, J. F. (2017). Functional roles of astrocyte calcium elevations: from synapses to behavior. Front. Cell Neurosci. 11:427. doi: $10.3389 /$ fncel.2017.00427

Guthrie, P. B., Knappenberger, J., Segal, M., Bennett, M. V., Charles, A. C., and Kater, S. B. (1999). ATP released from astrocytes mediates glial calcium waves. J. Neurosci. 19, 520-528. doi: 10.1523/jneurosci.19-02-00520.1999

Guttenplan, K. A., Weigel, M. K., Adler, D. I., Couthouis, J., Liddelow, S. A., Gitler, A. D., et al. (2020). Knockout of reactive astrocyte activating factors slows disease progression in an ALS mouse model. Nat. Commun. 11:3753. doi: 10.1038/s41467-020-17514-9

Halassa, M. M., Florian, C., Fellin, T., Munoz, J. R., Lee, S. Y., Abel, T., et al. (2009). Astrocytic modulation of sleep homeostasis and cognitive consequences of sleep loss. Neuron 61, 213-219. doi: 10.1016/j.neuron.2008.11.024

Harada, K., Kamiya, T., and Tsuboi, T. (2015). Gliotransmitter release from astrocytes: functional, developmental, and pathological implications in the brain. Front. Neurosci. 9:499. doi: 10.3389/fnins.2015.00499

Haydon, P. G. (2017). Astrocytes and the modulation of sleep. Curr. Opin. Neurobiol. 44, 28-33. doi: 10.1016/j.conb.2017.02.008

Henneberger, C., and Rusakov, D. A. (2010). Synaptic plasticity and Ca2+ signalling in astrocytes. Neuron Glia Biol. 6, 141-146. doi: 10.1017/S1740925X1000 0153

Horvath, T. L., Sarman, B., Garcia-Caceres, C., Enriori, P. J., Sotonyi, P., Shanabrough, M., et al. (2010). Synaptic input organization of the melanocortin system predicts diet-induced hypothalamic reactive gliosis and obesity. Proc. Natl. Acad. Sci. U.S.A. 107, 14875-14880. doi: 10.1073/pnas.1004282107

Hsuchou, H., Pan, W., Barnes, M. J., and Kastin, A. J. (2009). Leptin receptor mRNA in rat brain astrocytes. Peptides 30, 2275-2280. doi: 10.1016/j.peptides. 2009.08.023

Iwai, Y., Ozawa, K., Yahagi, K., Mishima, T., Akther, S., Vo, C. T., et al. (2021). Transient astrocytic Gq signaling underlies remote memory enhancement. Front. Neural. Circuits 15:658343. doi: 10.3389/fncir.2021.658343

Jackson, T. R., Patterson, S. I., Thastrup, O., and Hanley, M. R. (1988). A novel tumour promoter, thapsigargin, transiently increases cytoplasmic free $\mathrm{Ca} 2+$ without generation of inositol phosphates in NG115-401L neuronal cells. Biochem. J. 253, 81-86. doi: 10.1042/bj2530081

Jones, M. E., Paniccia, J. E., Lebonville, C. L., Reissner, K. J., and Lysle, D. T. (2018). Chemogenetic manipulation of dorsal hippocampal astrocytes protects against the development of stress-enhanced fear learning. Neuroscience 388, 45-56. doi: 10.1016/j.neuroscience.2018.07.015

Jourdain, P., Bergersen, L. H., Bhaukaurally, K., Bezzi, P., Santello, M., Domercq, M., et al. (2007). Glutamate exocytosis from astrocytes controls synaptic strength. Nat. Neurosci. 10, 331-339. doi: 10.1038/nn1849

Kang, S., Hong, S. I., Lee, J., Peyton, L., Baker, M., Choi, S., et al. (2020). Activation of astrocytes in the dorsomedial striatum facilitates transition from habitual to goal-directed reward-seeking behavior. Biol. Psychiatry 88, 797-808. doi: 10.1016/j.biopsych.2020.04.023

Khakh, B. S., and McCarthy, K. D. (2015). Astrocyte calcium signaling: from observations to functions and the challenges therein. Cold Spring Harb. Perspect. Biol. 7:a020404. doi: 10.1101/cshperspect.a020404

Kim, J. G., Suyama, S., Koch, M., Jin, S., Argente-Arizon, P., Argente, J., et al. (2014). Leptin signaling in astrocytes regulates hypothalamic neuronal circuits and feeding. Nat. Neurosci. 17, 908-910. doi: 10.1038/nn.3725

Klinzing, J. G., Niethard, N., and Born, J. (2019). Mechanisms of systems memory consolidation during sleep. Nat. Neurosci. 22, 1598-1610. doi: 10.1038/s41593019-0467-3

Kofuji, P., and Araque, A. (2021). G-Protein-coupled receptors in astrocyte-neuron communication. Neuroscience 456, 71-84. doi: 10.1016/j.neuroscience.2020. 03.025

Kol, A., Adamsky, A., Groysman, M., Kreisel, T., London, M., and Goshen, I. (2020). Astrocytes contribute to remote memory formation by modulating hippocampal-cortical communication during learning. Nat. Neurosci. 23, 12291239. doi: 10.1038/s41593-020-0679-6

Krashes, M. J., Koda, S., Ye, C., Rogan, S. C., Adams, A. C., Cusher, D. S., et al. (2011). Rapid, reversible activation of AgRP neurons drives feeding behavior in mice. J. Clin. Invest. 121, 1424-1428. doi: 10.1172/JCI46229

Kwak, H., Koh, W., Kim, S., Song, K., Shin, J. I., Lee, J. M., et al. (2020). Astrocytes control sensory acuity via tonic inhibition in the thalamus. Neuron 108, 691.e610-706.e610. doi: 10.1016/j.neuron.2020.08.013
Landis, D. M., and Reese, T. S. (1982). Regional organization of astrocytic membranes in cerebellar cortex. Neuroscience 7, 937-950. doi: 10.1016/03064522(82)90053-7

Lee, H. S., Ghetti, A., Pinto-Duarte, A., Wang, X., Dziewczapolski, G., Galimi, F., et al. (2014). Astrocytes contribute to gamma oscillations and recognition memory. Proc. Natl. Acad. Sci. U.S.A. 111, E3343-E3352. doi: 10.1073/pnas. 1410893111

Lee, Y., Messing, A., Su, M., and Brenner, M. (2008). GFAP promoter elements required for region-specific and astrocyte-specific expression. Glia 56, 481-493. doi: 10.1002/glia.20622

Leopold, A. V., Shcherbakova, D. M., and Verkhusha, V. V. (2019). Fluorescent biosensors for neurotransmission and neuromodulation: engineering and applications. Front. Cell Neurosci. 13:474. doi: 10.3389/fncel.2019.00474

Lines, J., Martin, E. D., Kofuji, P., Aguilar, J., and Araque, A. (2020). Astrocytes modulate sensory-evoked neuronal network activity. Nat. Commun. 11:3689. doi: 10.1038/s41467-020-17536-3

Looger, L. L., and Griesbeck, O. (2012). Genetically encoded neural activity indicators. Curr. Opin. Neurobiol. 22, 18-23. doi: 10.1016/j.conb.2011.10.024

MacDonald, A. J., Holmes, F. E., Beall, C., Pickering, A. E., and Ellacott, K. L. J. (2020). Regulation of food intake by astrocytes in the brainstem dorsal vagal complex. Glia 68, 1241-1254. doi: 10.1002/glia.23774

Maquet, P. (2001). The role of sleep in learning and memory. Science 294, $1048-$ 1052. doi: $10.1126 /$ science. 1062856

Maren, S. (2008). Pavlovian fear conditioning as a behavioral assay for hippocampus and amygdala function: cautions and caveats. Eur. J. Neurosci. 28, 1661-1666. doi: 10.1111/j.1460-9568.2008.06485.x

Mariotti, L., Losi, G., Sessolo, M., Marcon, I., and Carmignoto, G. (2016). The inhibitory neurotransmitter GABA evokes long-lasting $\mathrm{Ca}(2+)$ oscillations in cortical astrocytes. Glia 64, 363-373. doi: 10.1002/glia.22933

Martin-Fernandez, M., Jamison, S., Robin, L. M., Zhao, Z., Martin, E. D., Aguilar, J., et al. (2017). Synapse-specific astrocyte gating of amygdala-related behavior. Nat. Neurosci. 20, 1540-1548. doi: 10.1038/nn.4649

Mathis, A., Mamidanna, P., Cury, K. M., Abe, T., Murthy, V. N., Mathis, M. W., et al. (2018). DeepLabCut: markerless pose estimation of user-defined body parts with deep learning. Nat. Neurosci. 21, 1281-1289. doi: 10.1038/s41593018-0209-y

Mazur, A., Bills, E. H., DeSchepper, K. M., Williamson, J. C., Henderson, B. J., and Risher, W. C. (2021). Astrocyte-derived thrombospondin induces cortical synaptogenesis in a sex-specific manner. eNeuro 8, ENEURO.0014ENEURO.21. doi: 10.1523/ENEURO.0014-21.2021

McNeill, J., Rudyk, C., Hildebrand, M. E., and Salmaso, N. (2021). Ion channels and electrophysiological properties of astrocytes: implications for emergent stimulation technologies. Front. Cell Neurosci. 15:644126. doi: 10.3389/fncel. 2021.644126

Mederos, S., Hernandez-Vivanco, A., Ramirez-Franco, J., Martin-Fernandez, M., Navarrete, M., Yang, A., et al. (2019). Melanopsin for precise optogenetic activation of astrocyte-neuron networks. Glia 67, 915-934. doi: 10.1002/glia. 23580

Mederos, S., Sanchez-Puelles, C., Esparza, J., Valero, M., Ponomarenko, A., and Perea, G. (2021). GABAergic signaling to astrocytes in the prefrontal cortex sustains goal-directed behaviors. Nat. Neurosci. 24, 82-92. doi: 10.1038/s41593020-00752-X

Mu, Y., Bennett, D. V., Rubinov, M., Narayan, S., Yang, C. T., Tanimoto, M., et al. (2019). Glia accumulate evidence that actions are futile and suppress unsuccessful behavior. Cell 178, 27.e19-43.e19. doi: 10.1016/j.cell.2019.05.050

Murphy-Royal, C., Dupuis, J., Groc, L., and Oliet, S. H. R. (2017). Astroglial glutamate transporters in the brain: regulating neurotransmitter homeostasis and synaptic transmission. J. Neurosci. Res. 95, 2140-2151. doi: 10.1002/jnr. 24029

Nagai, J., Yu, X., Papouin, T., Cheong, E., Freeman, M. R., Monk, K. R., et al. (2021a). Behaviorally consequential astrocytic regulation of neural circuits. Neuron 109, 576-596. doi: 10.1016/j.neuron.2020.12.008

Nagai, J., Bellafard, A., Qu, Z., Yu, X., Ollivier, M., Gangwani, M. R., et al. (2021b). Specific and behaviorally consequential astrocyte Gq GPCR signaling attenuation in vivo with ibetaARK. Neuron 109, 2256.e2259-2274.e2259. doi: 10.1016/j.neuron.2021.05.023

Nagai, J., Rajbhandari, A. K., Gangwani, M. R., Hachisuka, A., Coppola, G., Masmanidis, S. C., et al. (2019). Hyperactivity with disrupted attention by 
activation of an astrocyte synaptogenic cue. Cell 177, 1280.e1220-1292.e1220. doi: 10.1016/j.cell.2019.03.019

Nam, M. H., Han, K. S., Lee, J., Won, W., Koh, W., Bae, J. Y., et al. (2019). Activation of astrocytic mu-opioid receptor causes conditioned place preference. Cell Rep. 28, 1154.e1155-1166.e1155. doi: 10.1016/j.celrep.2019.06.071

Navarrete, M., Perea, G., Maglio, L., Pastor, J., Garcia de Sola, R., and Araque, A. (2013). Astrocyte calcium signal and gliotransmission in human brain tissue. Cereb Cortex 23, 1240-1246. doi: 10.1093/cercor/bhs122

Nett, W. J., Oloff, S. H., and McCarthy, K. D. (2002). Hippocampal astrocytes in situ exhibit calcium oscillations that occur independent of neuronal activity. J. Neurophysiol. 87, 528-537. doi: 10.1152/jn.00268.2001

Neves, S. R., Ram, P. T., and Iyengar, R. (2002). G protein pathways. Science 296, 1636-1639. doi: 10.1126/science. 1071550

Nimmerjahn, A. (2009). Astrocytes going live: advances and challenges. J. Physiol. 587(Pt 8), 1639-1647. doi: 10.1113/jphysiol.2008.167171

Nimmerjahn, A., Mukamel, E. A., and Schnitzer, M. J. (2009). Motor behavior activates Bergmann glial networks. Neuron 62, 400-412. doi: 10.1016/j.neuron. 2009.03.019

Nwachukwu, K. N., Evans, W. A., Sides, T. R., Trevisani, C. P., Davis, A., and Marshall, S. A. (2021). Chemogenetic manipulation of astrocytic signaling in the basolateral amygdala reduces binge-like alcohol consumption in male mice. J. Neurosci. Res. 99, 1957-1972. doi: 10.1002/jnr.24841

Octeau, J. C., Gangwani, M. R., Allam, S. L., Tran, D., Huang, S., HoangTrong, T. M., et al. (2019). Transient, consequential increases in extracellular potassium ions accompany Channelrhodopsin2 excitation. Cell Rep. 27, 2249.e2247-2261.e2247. doi: 10.1016/j.celrep.2019.04.078

Orr, A. G., Hsiao, E. C., Wang, M. M., Ho, K., Kim, D. H., Wang, X., et al. (2015). Astrocytic adenosine receptor A2A and Gs-coupled signaling regulate memory. Nat. Neurosci. 18, 423-434. doi: 10.1038/nn.3930

Padilla, S. L., Qiu, J., Soden, M. E., Sanz, E., Nestor, C. C., Barker, F. D., et al. (2016). Agouti-related peptide neural circuits mediate adaptive behaviors in the starved state. Nat. Neurosci. 19, 734-741. doi: 10.1038/nn.4274

Pan, J., Ma, N., Yu, B., Zhang, W., and Wan, J. (2020). Transcriptomic profiling of microglia and astrocytes throughout aging. J. Neuroinflamm. 17:97. doi: 10.1186/s12974-020-01774-9

Panatier, A., Theodosis, D. T., Mothet, J. P., Touquet, B., Pollegioni, L., Poulain, D. A., et al. (2006). Glia-derived D-serine controls NMDA receptor activity and synaptic memory. Cell 125, 775-784. doi: 10.1016/j.cell.2006.02.051

Pascual, O., Casper, K. B., Kubera, C., Zhang, J., Revilla-Sanchez, R., Sul, J. Y., et al. (2005). Astrocytic purinergic signaling coordinates synaptic networks. Science 310, 113-116. doi: 10.1126/science.1116916

Paukert, M., Agarwal, A., Cha, J., Doze, V. A., Kang, J. U., and Bergles, D. E. (2014). Norepinephrine controls astroglial responsiveness to local circuit activity. Neuron 82, 1263-1270. doi: 10.1016/j.neuron.2014.04.038

Pelluru, D., Konadhode, R. R., Bhat, N. R., and Shiromani, P. J. (2016). Optogenetic stimulation of astrocytes in the posterior hypothalamus increases sleep at night in C57BL/6J mice. Eur. J. Neurosci. 43, 1298-1306. doi: 10.1111/ejn.13074

Perea, G., Yang, A., Boyden, E. S., and Sur, M. (2014). Optogenetic astrocyte activation modulates response selectivity of visual cortex neurons in vivo. Nat. Commun. 5:3262. doi: 10.1038/ncomms4262

Petravicz, J., Fiacco, T. A., and McCarthy, K. D. (2008). Loss of IP3 receptordependent $\mathrm{Ca} 2+$ increases in hippocampal astrocytes does not affect baseline CA1 pyramidal neuron synaptic activity. J. Neurosci. 28, 4967-4973. doi: 10. 1523/JNEUROSCI.5572-07.2008

Pinto-Duarte, A., Roberts, A. J., Ouyang, K., and Sejnowski, T. J. (2019). Impairments in remote memory caused by the lack of Type 2 IP3 receptors. Glia 67, 1976-1989. doi: 10.1002/glia.23679

Porter, J. T., and McCarthy, K. D. (1997). Astrocytic neurotransmitter receptors in situ and in vivo. Prog. Neurobiol. 51, 439-455. doi: 10.1016/s0301-0082(96) 00068-8

Poskanzer, K. E., and Yuste, R. (2016). Astrocytes regulate cortical state switching in vivo. Proc. Natl. Acad. Sci. U.S.A. 113, E2675-E2684. doi: 10.1073/pnas. 1520759113

Prendergast, B. J., Onishi, K. G., and Zucker, I. (2014). Female mice liberated for inclusion in neuroscience and biomedical research. Neurosci. Biobehav. Rev. 40, 1-5. doi: 10.1016/j.neubiorev.2014.01.001

Rasmussen, R., Nicholas, E., Petersen, N. C., Dietz, A. G., Xu, Q., Sun, Q., et al. (2019). Cortex-wide changes in extracellular potassium ions parallel brain state transitions in awake behaving mice. Cell Rep. 28, 1182.e1184-1194.e1184. doi: 10.1016/j.celrep.2019.06.082

Ros, O., Baudet, S., Zagar, Y., Loulier, K., Roche, F., Couvet, S., et al. (2020). SpiCee: a genetic tool for subcellular and cell-specific calcium manipulation. Cell Rep. 32:107934. doi: 10.1016/j.celrep.2020.107934

Rose, C. R., and Ransom, B. R. (1997). Gap junctions equalize intracellular $\mathrm{Na}+$ concentration in astrocytes. Glia $20,299-307$. doi: $10.1002 /($ sici)10981136(199708)20:4<299::aid-glia3<3.0.co;2-1

Roth, B. L. (2016). DREADDs for neuroscientists. Neuron 89, 683-694. doi: 10. 1016/j.neuron.2016.01.040

Roth, R. H., and Ding, J. B. (2020). From neurons to cognition: technologies for precise recording of neural activity underlying behavior. BME Front. 2020:20. doi: 10.34133/2020/7190517

Rungta, R. L., Bernier, L. P., Dissing-Olesen, L., Groten, C. J., LeDue, J. M., Ko, R., et al. (2016). $\mathrm{Ca}(2+)$ transients in astrocyte fine processes occur via $\mathrm{Ca}(2+)$ influx in the adult mouse hippocampus. Glia 64, 2093-2103. doi: 10.1002/glia. 23042

Rurak, G. M., Simard, S., Charih, F., Van Geel, A., Stead, J., Woodside, B., et al. (2020). Translatomic database of cortical astroglia across male and female mouse development reveals two distinct developmental phenotypes. bioRxiv [Preprint]. doi: 10.1101/681684

Sancho, L., Contreras, M., and Allen, N. J. (2021). Glia as sculptors of synaptic plasticity. Neurosci. Res. 167, 17-29. doi: 10.1016/j.neures.2020.11.005

Santello, M., Toni, N., and Volterra, A. (2019). Astrocyte function from information processing to cognition and cognitive impairment. Nat. Neurosci. 22, 154-166. doi: 10.1038/s41593-018-0325-8

Sasaki, T., Beppu, K., Tanaka, K. F., Fukazawa, Y., Shigemoto, R., and Matsui, K. (2012). Application of an optogenetic byway for perturbing neuronal activity via glial photostimulation. Proc. Natl. Acad. Sci. U.S.A. 109, 20720-20725. doi: 10.1073/pnas.1213458109

Schummers, J., Yu, H., and Sur, M. (2008). Tuned responses of astrocytes and their influence on hemodynamic signals in the visual cortex. Science 320, 1638-1643. doi: 10.1126/science.1156120

Scofield, M. D., Boger, H. A., Smith, R. J., Li, H., Haydon, P. G., and Kalivas, P. W. (2015). Gq-DREADD selectively initiates glial glutamate release and inhibits cue-induced cocaine seeking. Biol. Psychiatry 78, 441-451. doi: 10. 1016/j.biopsych.2015.02.016

Shan, L., Zhang, T., Fan, K., Cai, W., and Liu, H. (2021). Astrocyte-neuron signaling in synaptogenesis. Front. Cell Dev. Biol. 9:680301. doi: 10.3389/fcell.2021. 680301

Shelkar, G. P., Liu, J., and Dravid, S. M. (2021). Astrocytic NMDA receptors in the basolateral amygdala contribute to facilitation of fear extinction. Int. J. Neuropsychopharmacol. doi: 10.1093/ijnp/py ab055[Epub ahead of print].

Shigetomi, E., Bowser, D. N., Sofroniew, M. V., and Khakh, B. S. (2008). Two forms of astrocyte calcium excitability have distinct effects on NMDA receptormediated slow inward currents in pyramidal neurons. J. Neurosci. 28, 66596663. doi: 10.1523/JNEUROSCI.1717-08.2008

Shigetomi, E., Bushong, E. A., Haustein, M. D., Tong, X., Jackson-Weaver, O., Kracun, S., et al. (2013). Imaging calcium microdomains within entire astrocyte territories and endfeet with GCaMPs expressed using adenoassociated viruses. J. Gen. Physiol. 141, 633-647. doi: 10.1085/jgp.20121 0949

Shigetomi, E., Kracun, S., Sofroniew, M. V., and Khakh, B. S. (2010). A genetically targeted optical sensor to monitor calcium signals in astrocyte processes. Nat. Neurosci. 13, 759-766. doi: 10.1038/nn.2557

Srinivasan, R., Lu, T. Y., Chai, H., Xu, J., Huang, B. S., Golshani, P., et al. (2016). New transgenic mouse lines for selectively targeting astrocytes and studying calcium signals in astrocyte processes in situ and in vivo. Neuron 92, 1181-1195. doi: 10.1016/j.neuron.2016.11.030

Stackhouse, T. L., and Mishra, A. (2021). Neurovascular coupling in development and disease: focus on astrocytes. Front. Cell Dev. Biol. 9:702832. doi: 10.3389/ fcell.2021.702832

Stickgold, R. (2005). Sleep-dependent memory consolidation. Nature 437, $1272-$ 1278. doi: $10.1038 /$ nature 04286

Suminaite, D., Lyons, D. A., and Livesey, M. R. (2019). Myelinated axon physiology and regulation of neural circuit function. Glia 67, 2050-2062. doi: 10.1002/glia. 23665 
Suzuki, A., Stern, S. A., Bozdagi, O., Huntley, G. W., Walker, R. H., Magistretti, P. J., et al. (2011). Astrocyte-neuron lactate transport is required for long-term memory formation. Cell 144, 810-823. doi: 10.1016/j.cell.2011. 02.018

Sweeney, P., Qi, Y., Xu, Z., and Yang, Y. (2016). Activation of hypothalamic astrocytes suppresses feeding without altering emotional states. Glia 64, $2263-$ 2273. doi: $10.1002 /$ glia. 23073

Swonger, A. K., and Rech, R. H. (1972). Serotonergic and cholinergic involvement in habituation of activity and spontaneous alternation of rats in a $\mathrm{Y}$ maze. J. Comp. Physiol. Psychol. 81, 509-522. doi: 10.1037/h0033690

Tosches, M. A. (2017). Developmental and genetic mechanisms of neural circuit evolution. Dev. Biol. 431, 16-25. doi: 10.1016/j.ydbio.2017.06.016

Tran, C. H. T., Peringod, G., and Gordon, G. R. (2018). Astrocytes integrate behavioral state and vascular signals during functional hyperemia. Neuron 100 , 1133.e1133-1148.e1133. doi: 10.1016/j.neuron.2018.09.045

Tsai, H. H., Li, H., Fuentealba, L. C., Molofsky, A. V., Taveira-Marques, R., Zhuang, H., et al. (2012). Regional astrocyte allocation regulates CNS synaptogenesis and repair. Science 337, 358-362. doi: 10.1126/science.1222381

Ung, K., Tepe, B., Pekarek, B., Arenkiel, B. R., and Deneen, B. (2020). Parallel astrocyte calcium signaling modulates olfactory bulb responses. J. Neurosci. Res. 98, 1605-1618. doi: 10.1002/jnr.24634

Vaidyanathan, T. V., Collard, M., Yokoyama, S., Reitman, M. E., and Poskanzer, K. E. (2021). Cortical astrocytes independently regulate sleep depth and duration via separate GPCR pathways. Elife 10:e63329. doi: 10.7554/eLife. 63329

Varela, L., Stutz, B., Song, J. E., Kim, J. G., Liu, Z. W., Gao, X. B., et al. (2021). Hunger-promoting AgRP neurons trigger an astrocyte-mediated feedforward autoactivation loop in mice. J. Clin. Invest. 131:e144239. doi: 10.1172/ JCI144239

Vorhees, C. V., and Williams, M. T. (2014). Assessing spatial learning and memory in rodents. ILAR J. 55, 310-332. doi: 10.1093/ilar/ilu013

Wahis, J., Hennes, M., Arckens, L., and Holt, M. G. (2021b). Star power: the emerging role of astrocytes as neuronal partners during cortical plasticity. Curr. Opin. Neurobiol. 67, 174-182. doi: 10.1016/j.conb.2020.12.001

Wahis, J., Baudon, A., Althammer, F., Kerspern, D., Goyon, S., Hagiwara, D., et al. (2021a). Astrocytes mediate the effect of oxytocin in the central amygdala on neuronal activity and affective states in rodents. Nat. Neurosci. 24, 529-541. doi: 10.1038/s41593-021-00800-0

Wang, X., Lou, N., Xu, Q., Tian, G. F., Peng, W. G., Han, X., et al. (2006). Astrocytic Ca2+ signaling evoked by sensory stimulation in vivo. Nat. Neurosci. 9, 816-823. doi: $10.1038 / \mathrm{nn} 1703$

Wang, Y., DelRosso, N. V., Vaidyanathan, T. V., Cahill, M. K., Reitman, M. E., Pittolo, S., et al. (2019). Accurate quantification of astrocyte and neurotransmitter fluorescence dynamics for single-cell and populationlevel physiology. Nat. Neurosci. 22, 1936-1944. doi: 10.1038/s41593-0190492-2

Werneburg, S., Feinberg, P. A., Johnson, K. M., and Schafer, D. P. (2017). A microglia-cytokine axis to modulate synaptic connectivity and function. Curr. Opin. Neurobiol. 47, 138-145. doi: 10.1016/j.conb.2017.10.002

Wiktorowska, L., Bilecki, W., Tertil, M., Kudla, L., Szumiec, L., Mackowiak, M., et al. (2021). Knockdown of the astrocytic glucocorticoid receptor in the central nucleus of the amygdala diminishes conditioned fear expression and anxiety. Behav. Brain Res. 402:113095. doi: 10.1016/j.bbr.2020.113095

Wilhelm, C. J., Hashimoto, J. G., Roberts, M. L., Bloom, S. H., Andrew, M. R., and Wiren, K. M. (2016). Astrocyte dysfunction induced by alcohol in females but not males. Brain Pathol. 26, 433-451. doi: 10.1111/bpa.12276

Wiltschko, A. B., Tsukahara, T., Zeine, A., Anyoha, R., Gillis, W. F., Markowitz, J. E., et al. (2020). Revealing the structure of pharmacobehavioral space through motion sequencing. Nat. Neurosci. 23, 1433-1443. doi: 10.1038/s41593-02000706-3

Wolf, A., Bauer, B., Abner, E. L., Ashkenazy-Frolinger, T., and Hartz, A. M. (2016). A comprehensive behavioral test battery to assess learning and memory in 129S6/Tg2576 mice. PLoS One 11:e0147733. doi: 10.1371/journal.pone. 0147733
Wolosker, H., Blackshaw, S., and Snyder, S. H. (1999). Serine racemase: a glial enzyme synthesizing $\mathrm{D}$-serine to regulate glutamate-N-methyl-D-aspartate neurotransmission. Proc. Natl. Acad. Sci. U.S.A. 96, 13409-13414. doi: 10.1073/ pnas.96.23.13409

Wright, C. L., Schwarz, J. S., Dean, S. L., and McCarthy, M. M. (2010). Cellular mechanisms of estradiol-mediated sexual differentiation of the brain. Trends Endocrinol. Metab. 21, 553-561. doi: 10.1016/j.tem.2010.05.004

Wu, Q., and Palmiter, R. D. (2011). GABAergic signaling by AgRP neurons prevents anorexia via a melanocortin-independent mechanism. Eur. J. Pharmacol. 660, 21-27. doi: 10.1016/j.ejphar.2010.10.110

Xie, L., Kang, H., Xu, Q., Chen, M. J., Liao, Y., Thiyagarajan, M., et al. (2013). Sleep drives metabolite clearance from the adult brain. Science 342, 373-377. doi: 10.1126/science.1241224

Xie, Y., Wang, T., Sun, G. Y., and Ding, S. (2010). Specific disruption of astrocytic $\mathrm{Ca} 2+$ signaling pathway in vivo by adeno-associated viral transduction. Neuroscience 170, 992-1003. doi: 10.1016/j.neuroscience.2010.08.034

Xin, W., and Chan, J. R. (2020). Myelin plasticity: sculpting circuits in learning and memory. Nat. Rev. Neurosci. 21, 682-694. doi: 10.1038/s41583-020-00379-8

Yamashita, A., Hamada, A., Suhara, Y., Kawabe, R., Yanase, M., Kuzumaki, N., et al. (2014). Astrocytic activation in the anterior cingulate cortex is critical for sleep disorder under neuropathic pain. Synapse 68, 235-247. doi: 10.1002/syn.21733

Yang, L., Qi, Y., and Yang, Y. (2015). Astrocytes control food intake by inhibiting AGRP neuron activity via adenosine A1 receptors. Cell Rep. 11, 798-807. doi: 10.1016/j.celrep.2015.04.002

Yang, Y., Ge, W., Chen, Y., Zhang, Z., Shen, W., Wu, C., et al. (2003). Contribution of astrocytes to hippocampal long-term potentiation through release of D-serine. Proc. Natl. Acad. Sci. U.S.A. 100, 15194-15199. doi: 10. 1073/pnas. 2431073100

Yirmiya, R., and Goshen, I. (2011). Immune modulation of learning, memory, neural plasticity and neurogenesis. Brain Behav. Immun. 25, 181-213. doi: 10.1016/j.bbi.2010.10.015

Yu, X., Nagai, J., and Khakh, B. S. (2020). Improved tools to study astrocytes. Nat. Rev. Neurosci. 21, 121-138. doi: 10.1038/s41583-020-0264-8

Yu, X., Taylor, A. M. W., Nagai, J., Golshani, P., Evans, C. J., Coppola, G., et al. (2018). Reducing astrocyte calcium signaling in vivo alters striatal microcircuits and causes repetitive behavior. Neuron 99, 1170.e1179-1187.e1179. doi: 10. 1016/j.neuron.2018.08.015

Zhang, Y., Chen, K., Sloan, S. A., Bennett, M. L., Scholze, A. R., O'Keeffe, S., et al. (2014). An RNA-sequencing transcriptome and splicing database of glia, neurons, and vascular cells of the cerebral cortex. J. Neurosci. 34, 11929-11947. doi: 10.1523/JNEUROSCI.1860-14.2014

Zhou, Z., Okamoto, K., Onodera, J., Hiragi, T., Andoh, M., Ikawa, M., et al. (2021). Astrocytic cAMP modulates memory via synaptic plasticity. Proc. Natl. Acad. Sci. U.S.A. 118:e2016584118. doi: 10.1073/pnas.2016584118

Zorec, R., Araque, A., Carmignoto, G., Haydon, P. G., Verkhratsky, A., and Parpura, V. (2012). Astroglial excitability and gliotransmission: an appraisal of $\mathrm{Ca} 2+$ as a signalling route. ASN Neuro 4:e00080. doi: 10.1042/AN20110061

Conflict of Interest: The authors declare that the research was conducted in the absence of any commercial or financial relationships that could be construed as a potential conflict of interest.

Publisher's Note: All claims expressed in this article are solely those of the authors and do not necessarily represent those of their affiliated organizations, or those of the publisher, the editors and the reviewers. Any product that may be evaluated in this article, or claim that may be made by its manufacturer, is not guaranteed or endorsed by the publisher.

Copyright $(2022$ Lyon and Allen. This is an open-access article distributed under the terms of the Creative Commons Attribution License (CC BY). The use, distribution or reproduction in other forums is permitted, provided the original author(s) and the copyright owner(s) are credited and that the original publication in this journal is cited, in accordance with accepted academic practice. No use, distribution or reproduction is permitted which does not comply with these terms. 